\title{
TTR
}

Traduction, terminologie, rédaction

\section{Antoine Berman. L'Âge de la traduction. "La tâche du traducteur " de Walter Benjamin, un commentaire. Texte établi par Isabelle Berman avec la collaboration de Valentina Sommella. Saint-Denis, Presses Universitaires de Vincennes, coll. " Intempestives », 2008}

\section{Laurent Lamy}

Volume 23, numéro 1, 1er semestre 2010

Rencontres est-ouest

East-West Encounters

URI : https://id.erudit.org/iderudit/044937ar

DOI : https://doi.org/10.7202/044937ar

Aller au sommaire du numéro

Éditeur(s)

Association canadienne de traductologie

ISSN

0835-8443 (imprimé)

1708-2188 (numérique)

Découvrir la revue

Citer ce compte rendu

Lamy, L. (2010). Compte rendu de [Antoine Berman. L'Âge de la traduction. " La tâche du traducteur » de Walter Benjamin, un commentaire. Texte établi par Isabelle Berman avec la collaboration de Valentina Sommella. Saint-Denis, Presses Universitaires de Vincennes, coll. «Intempestives », 2008]. TTR, 23(1), 210-258. https://doi.org/10.7202/044937ar

Tous droits réservés @ TTR: traduction, terminologie, rédaction — Les auteurs, 2010
Ce document est protégé par la loi sur le droit d'auteur. L'utilisation des services d'Érudit (y compris la reproduction) est assujettie à sa politique d'utilisation que vous pouvez consulter en ligne.

https://apropos.erudit.org/fr/usagers/politique-dutilisation/ 
Antoine Berman. L'Âge de la traduction. "La tâche du traducteur " de Walter Benjamin, un commentaire. Texte établi par Isabelle Berman avec la collaboration de Valentina Sommella. Saint-Denis, Presses Universitaires de Vincennes, coll. «Intempestives ", 2008 1 .

Tu pourvoiras l'arche (tēvah) d'une baie lumineuse.

(Genèse 6, 16)

Tu pourvoiras chaque mot (tèvah) d'une baie lumineuse, afin qu'il brille comme soleil en plein midi. (Interprétation kabbalistique de Genèse 6, 16)

Kommentar und Übersetzung verhalten sich zum Text wie Stil und Mimesis zur Natur: dasselbe Phänomen unter verschiedenen Betrachtungsweisen. Am Baum des heiligens Textes sind Beide nur die ewig rauschenden Blätter, am Baum des profanen die rechtzeitig fallenden früchte.

Tel un voile aux amples plis drapant la mémoire d'Antoine Berman : l'amour de la lettre comme suprême ornement de la vie

Le commentaire et la traduction ont avec le texte les mêmes rapports que le style et la mimésis avec la nature : le même phénomène considéré de manière différente. Sur l'arbre du texte sacré, ils ne sont tous les deux que les feuilles qui bruissent éternellement; sur l'arbre du texte profane, les fruits qui tombent le moment venu (rechtzeitig). (Walter Benjamin, 1972, p. 92)

Avouons-le d'entrée de jeu : il est des textes que nous abordons avec un surcroît irrépressible de fébrilité, comme si une attente insoupçonnée, mais vaguement pressentie, nous y soudait déjà, comme une épreuve redoutée, car le vertige anticipé, dédale de doutes chevauchant des spirales d'épiphanies livrées sous forme de "lectures tangentes ", est la seule clef qui nous puisse bailler accès à l'inédit.

1 Cité désormais $A T$. 
L'inédit en question ici est le séminaire qu'Antoine Berman a consacré à "La tâche du traducteur " de Walter Benjamin dans le cadre du Collège international de philosophie à la session d'hiver 1984-1985.

D'abord, une remarque sur l'intendance très soignée du processus éditorial, qui a immunisé les traces de ce séminaire, dans ses versants oral et écrit, de toute incurie. La patiente maturation $\mathrm{du}$ manuscrit et des bandes sonores par les soins d'Isabelle Berman et d'un groupe d'amis dévoués à la perpétuation du legs de Berman, dont sa proche collaboratrice Valentina Sommella, nous a acheminé un texte d'une facture très aboutie et d'une parfaite lisibilité. Pareilles qualités, empreintes de sollicitude, prévalent aussi bien dans son rendu que dans sa logique, dont la division obéit à l'échelonnement du support matériel sur lequel Berman préparait ses prestations : des cahiers. Un texte inédit, intercalé entre la Note éditoriale d'Isabelle Berman et le corps du séminaire proprement dit, livré sous forme de post mortem retraçant à tire-d'aile la maîtrise d'œuvre à laquelle Berman s'était employé au Collège international de philosophie, campe les divers états du questionnement jalonnant l'évolution de ces séminaires. La clarté est partout au rendez-vous (AT, pp. 9-13).

\section{Ouverture}

\section{Cahier 1}

La déclaration inaugurale de Berman est péremptoire et sans équivoque, comme si elle coulait de source : l'essai de Benjamin est le texte central du $\mathrm{XX}^{\mathrm{e}}$ siècle sur la traduction. Il est indépassable, que l'on y adhère ou que l'on se dresse contre lui. Il est l'épicentre, le point névralgique où convergent les divers affluents de l'expérience allemande de la traduction, de l'éclosion météorique de la vaste spéculation développée par l'aréopage des Romantiques évoluant autour de l'Athenäum à Iéna jusqu'aux ruminations crépusculaires de Stefan George.

La radicalité du diagnostic de Berman, non moins que celle qui émaille les veines du texte de Benjamin, sollicite un genre, une modalité dans l'approche, celle du « commentaire », qui est ici appelé à reprendre du métier. L'autonomisation du discours 
critique avait progressivement mis sous le boisseau cette tradition très ancienne du commentaire qui nourrit un lien d'essence avec l'exercice de la traduction.

J'ouvre ici une brève parenthèse en appui à l'option de Berman : si, par exemple, la pratique du commentaire fut fort en vogue chez les doctes médiévaux, c'est que l'impératif de traduction les tenait constamment à pied d'œuvre face à la masse critique des corpus grec et hellénistique hérités par le relais arabomusulman et la médiation syriaque depuis Byzance. Qui plus est, ils étaient pour ainsi dire condamnés à l'émulation à ce chapitre, puisque l'art du commentaire avait déjà atteint un niveau inégalé, quasi paradigmatique, monumental, dans les grandes fresques spéculatives léguées par Averroës (ibn Rushd, 1126-1198), Avicenne (ibn Sīnā, 980-1037) et Abū Nasr al-Farābī (872-950) qui, s'affairant autour des canons grecs, pistaient à la ligne et au mot près les multiples cas de figures enchâssées dans la « lettre " au gré de traductions toujours au banc d'essai.

Je me permets aussi de préciser que si traduction et commentaire ont partie liée, c'est que leur gémellité est inhérente à la distance éprouvée dans l'abord de tout texte étranger, dans la mesure où l'ouverture, la sollicitude ou la visée anticipée de la langue ou de la culture-hôte est toujours affectée d'un coefficient d'asymétrie incorrigible dont l'écart ou l'angle de déférence ménage les avenues du commentaire, le sollicite comme tel. Comme le stipule Berman, « tout commentaire d'un texte étranger comporte un travail de traduction. À la limite, est traduction " (AT, p. 18).

Or que l'essai de Benjamin ne puisse s'ouvrir qu'à un commentaire signale en creux ce lien d'essence, mais signifie aussi que cet exercice singulier qu'est le commentaire ne peut exister qu'à partir de l'original, de "l'œuvre-dans-sa-langue » ( $A T$, p. 19). Le commentaire ne s'adresse pas au texte traduit, puisqu'il appartient au processus même de la traduction ou, comme l'écrit Berman, "se déploie dans la dimension de la traduction " (AT, p. 19). Commenter un texte traduit satelliserait la lettre au seul profit du sens, alors que le commentaire est essentiellement commentaire-de-la-lettre. En revanche, au commentaire de la lettre 
peut s'articuler une analyse de sa traduction ou de ses traductions : c'est à la faveur de cette articulation bi-univoque " qui permet d'éclairer multiplement le texte, et de rapprocher le commentaire de son essence traductive " ( $A T$, p. 20) que l'épreuve de la traduction se révèle comme momentum critique de l'œuvre, dont la convergence éclate au moment opportun, rechtzeitig, comme l'écrit Benjamin dans Einbahnstrasse.

Ici je schématise le propos de Berman : le commentaire voué à la « lettre » de l'original avalise l'analyse de la traduction qui en est proposée, laquelle en retour déploie sur un axe longitudinal ce qui s'est ouvert dans la plongée latitudinale où l'on s'enquiert des ressorts de la lettre, qui est l'épreuve de l'étranger proprement dite. Le commentaire se dédouble en quelque sorte, je dirais même qu'il est polarisé, en vertu de la traduisibilité inhérente à l'œuvre, par l'éclosion du momentum critique qui doit en épuiser l'épiphanie et libérer les potentialités latentes à la faveur d'une retraduction qu'il sollicite à son insu, de sorte qu'il est « fatalement amené, ensuite, à re-traduire, ou plutôt à traduire sur le mode de la re-traduction, c'est-à-dire sur le mode le plus critique, le plus accompli de la traduction» $(A T$, p. 20).

Si je me suis attardé à cette considération liminaire, c'est que sa prise en compte nous donne le ton et nous permet de saisir l'enjeu et la portée de l'exercice auquel Berman compte se livrer, non moins que la teneur du texte qui sollicite cet exercice et qui impose sa lettre : je dirais : l'amour de la lettre.

J'en profite aussi pour corriger une perception répandue qui est presque devenue un poncif : d'aucuns s'entendent en effet pour cantonner Berman dans une posture sourcière. Ils peuvent remballer : ce dernier n'est pas davantage sourcier que cibliste, mais un pédagogue d'une remarquable constance qui a toujours souci d'opérer les distinctions préalables à l'exercice de la réflexion. Sa probité ne l'est pas moins, par exemple lorsqu'il refuse de vilipender la traduction proposée par Maurice de Gandillac, qui a littéralement initié, dès les années soixante, la chaîne des translations - commentaire, traduction, analyse, retraduction autour de l'essai de Benjamin. En fait, la traduction de Gandillac "présente les défaillances structurelles de toute première traduction" 
(AT, p. 20). Bref, l'épreuve de la traduction fournit le momentum critique pour la mise en œuvre du commentaire, qui est toujours, comme le dit Berman, «commentaire de commentaire " ( $A T$, p. 20). C'est ce que je me propose de faire ici. En espérant, à l'instar de Berman, que mon intervention sera rechtzeitig, opportune et porteuse de fruits prêts à tomber de «l'arbre du texte profane».

L'un des propos avoués de Benjamin, mais jamais réalisé dans toute l'ampleur qu'il anticipait, est l'élaboration d'une " métaphysique du langage ». La traduction en sera la clef de voûte, y occupant à tout le moins une place beaucoup plus prépondérante que celle, s'il en est, qui lui fut dévolue dans les philosophies traditionnelles du langage. Le postulat fondamental de cette "métaphysique du langage » est le refus net, catégorique, de ravaler l'exercice du langage au simple statut de moyen de communication ou à un système de signes. Pour Benjamin, le langage est un médium, un " milieu ». Comme l'écrit Berman, "le langage est le milieu de toutes les communications, mais n'est pas communication lui-même. Ce médium n’est pas indifférencié : il contient des "zones" plus ou moins denses, et le passage d'une zone moins dense à une zone plus dense, c'est la traduction » ( $A T$, p. 23).

Cette sensibilité toute particulière au langage comme « milieu », sinon comme « habitat » est une constante de la pensée allemande ${ }^{2}$. Berman cite Luther, Grimm, Heidegger et Hamann, notamment, qui en fut l'un des plus ardents zélateurs (voir Lamy, 2007, pp.34-44). J'ajouterais à cette courte liste le nom de Ludwig Wittgenstein, à qui répugnait toute forme d'instrumentalisation

2 Pour une critique fort bien argumentée, mais contestable de mon point de vue, voir l'essai de Cristina Lafont (2002); au relativisme présumé de ce courant de pensée, que l'on peut faire remonter à Hamann et Herder, joserais même prononcer le nom de Jacob Boehme, en passant par Humboldt, pour s'étendre jusqu'à Benjamin, Heidegger et Gadamer, Cristina Lafont oppose sa propre version de la " théorie de l'agir communicationnel "(Theorie des kommunicativen Handelns) élaborée par Jürgen Habermas. 
de l'exercice du langage, qu'il concevait plutôt comme une « forme de vie $»-$ ein Lebensform ${ }^{3}$.

La " métaphysique du langage » de Benjamin est chevillée par une notion centrale, celle de reine Sprache, de "langue pure " qui, comme le note Berman, est la corrélation exacte de l'expression kantienne "Raison pure ", reine Vernunft (AT, p. 24). Rompu aux arcanes de l'analytique transcendantale de Kant, Benjamin y déplore l'absence de quelque considération expresse sur la stratification foncière de toute connaissance, son soutènement dans les diverses couches expressives du langage. Influencé par Hamann, il y voit une carence majeure de l'architectonique kantienne, car il n'est d'expérience humaine qui puisse être réfléchie, donc faire l'objet d'un gain cognitif, sans passer par le filtre du langage dont chacune des déclinaisons historiques, les vernaculaires, porte à l'état de vestige des éclats de la reine Sprache.

Cette reine Sprache n'est pas qu'une vague réminiscence de quelque langue idyllique, adamique ou autre, qui aurait précédé la dissémination des idiomes après Babel, mais la teneur de vérité incarnée dans le "devenir des langues » et qui ne transparaît qu'à la faveur d'éphémères épiphanies, en un point de tangence furtive dont l'aire de jeu ménagée par l'exercice de la traduction constitue en quelque sorte le plan d'effraction, le momentum critique qui agit à l'instar d'un " révélateur " graphique où le symbolisant devient le symbolisé. Benjamin emboîte donc le pas, dans la foulée de Hamann et de Herder, pour projeter au tout premier plan le rôle éminemment formateur du langage comme dépositaire de la vérité et comme matrice des diverses formes qu'elle emprunte au gré de l'histoire, et ce non seulement comme véhicule de la teneur de vérité mais comme sa " matière première ", qui est appelée à devenir "forme en acte " dans la traduction. Derechef, je schématise, bref je condense et amplifie à la fois le propos de Berman, en fonction de ma connaissance de la pensée de Benjamin et de l'Aufgabe dont je viens de restituer

$3 \mathrm{Au} \S 19$ de ses Recherches philosophiques, Wittgenstein écrit, en effet, que « se représenter un langage, c'est se représenter une forme de vie eine Sprache vorstellen heißt, sich eine Lebensform vorstelle » (1960, p. 296). 
le noyau. C'est là l'apanage du commentaire, du moins tel que je le conçois. Et de Berman je pourrais dire ce qu'il affirme luimême de Benjamin : "Défendre la langue comme médium fondamental de l'expérience et de l'existence humaine était pour lui un impératif catégorique » $(A T$, p. 24).

Berman dégage ensuite cinq traits caractéristiques de la pensée de Benjamin. D'abord, le torse et le fragment. C'est le principe même de son écriture, où la forme fragmentée n'a rien d'un étiolement, d'une dislocation, mais cherche plutôt à fouir l'anfractuosité, l'interstice, la brisure, pour en tirer des esquisses définitives (AT, p. 25), qui tissent des constellations de formes symboliques au sein desquelles gravitent des ébauches d'une grande acuité et d'une parfaite concision, mais qui ne portent l'empreinte d'aucun système, à l'image de la ligne de faille que ne laisse de fouir une post-modernité métisse qui n'est autre que la constante reconfiguration du baroque généralisé dans la percolation parfois paroxystique des débris de l'histoire. Dans son étude sur Les affinités électives de Goethe, Benjamin écrit : " N'achève l'œuvre que d'abord ce qui la brise, pour faire d'elle une œuvre morcelée, un fragment du vrai monde, le débris d'un symbole »(1971, p. 234; 2000, pp. 274-395). Berman nous rappelle que « débris d'un symbole » est l'expression par laquelle Benjamin définit les langues naturelles dans 1'Aufgabe.

J'ouvre ici une parenthèse. L'écriture de Benjamin, loin d'être rhapsodique, obéit à ce principe : elle procède par touches chirurgicales, procédé qui s'apparente au regard micrologique mis en œuvre chez Adorno, pour ainsi percer au cœur d'un réseau souterrain, clandestin, d'« affinités électives » qu'il porte à découvert en reliant les aspects les plus prosaïques de la culture matérielle aux courants d'idées les plus abstraites qui irriguent la courbe du destin scellant la catastrophe anticipée qui allait voir l'Europe s'engouffrer dans les hécatombes que l'on sait et, ultimement, dans le mutisme impénétrable planant sur les camps de la mort. Ce qui nous est donné à contempler, ce sont les débris accumulés par le souffle violent du Progrès qui amoncelle « ruines sur ruines » précipitées aux pieds de l'Ange de l'Histoire, tel que nous le présente la vision élaborée par Benjamin dans sa IX ${ }^{\mathrm{e}}$ Thèse sur le concept d'histoire (2000b, p. 434). Je ne pousse 
pas plus loin mon analyse sur ce point pour ne pas égarer le fil de ma discussion avec le texte de Berman, sauf pour spécifier que j’y perçois aussi un principe de condensation optimale, de fulgurance dans l'éclat brisé qui sidère et pénètre au cœur de la réalité où la teneur de vérité des phénomènes transparaît l'espace d'un instant. C'est précisément ce que le traducteur doit activer, ce point de tangence furtive où se profile l'épiphanie de la reine Sprache. C'est aussi la seule façon de pallier la perte de l'aura qui a déserté les formes plastiques mais qui nétait en fin de compte, je le crois, que le préambule à une décanonisation accélérée des parangons de l'esthétique au profit d'une théorie critique célébrant l'inachèvement de l'œuvre, sa fragmentation stellaire transie par la force de l'ironie et la pointe vitriolée du Witz, sous l'ombre portée du nihilisme rompu à l'aveu de finitude, nous déportant au seuil de ce que d'aucuns ont désigné comme l'« ère du soupçon ». Pareillement, l'anticipation de cette reine Sprache, nous le verrons, n'est conçue par Benjamin que sur un mode tangentiel, à l'instar d'une "faible force messianique " qui serait appelée à libérer les harmoniques nouant de secrètes affinités entre les langues et qui seraient disséminées à l'état de germe dans leurs indénombrables modulations vernaculaires.

Ce qui nous amène à un second trait, complémentaire du premier : le divers et le même. L'échelonnement passablement hétéroclite, prolifique, des écrits de Benjamin affiche, comme le note Berman, une " absolue diversité » $(A T$, p. 26). Son écriture mobilise un spectre indéfini de thèmes et de topiques, associés tantôt à des artéfacts d'une déconcertante banalité, tantôt à l'articulation des notions les plus abstraites, enchevêtrant les divers plans et niveaux de réalité avec une singulière souplesse. En dépit de cette insaturable diversité, sa pensée se meut toujours dans le même élément, une maîtrise consommée de l'art dialectique, assortie à une aisance sans pareille pour ce qui est de naviguer dans l'amplitude hyperbolique entre la denrée concrète, la concrétude de la culture matérielle, qui est son terreau de prédilection, et un maniement tout aussi affine de la conceptualité la plus rigoureuse et parfois passablement sibylline.

Ce qui appelle déjà un troisième trait caractéristique de sa pensée : l'alliance précoce entre jeunesse et maturité. Auteur 
d'une étonnante maturité, Benjamin était très sensible au microcosme de l'enfance, dont il palpait les émois et scrutait la symbolique latente avec la sobriété d'une pensée très mûre. Il a généré l'essentiel de son œuvre entre 20 et 40 ans, et le fuseau de concepts qu'il a dégagé dans son essai sur Hölderlin, rédigé alors qu'il n'avait que 22 ans, transcende de très loin les fruits de la réflexion de la critique la plus moderne. Bien que Berman n'en touche mot, j'estime personnellement que c'est là le signe d'un tempérament saturnien, évoluant sous la coupe d'une melancholia métaphysique, comme d'ailleurs l'a fort bien intuitionné Susan Sontag dans son superbe essai Under the Sign of Saturn (2002, pp. 109-135).

Un quatrième trait est le rapport analogique entre concept et image. Comme le note Berman, "la pensée de Benjamin se déploie dans une dimension d'extrême conceptualité » (AT, p. 27). À cette maestria fulgurante qui projette sa pensée dans la sphère conceptuelle est assorti un répertoire idiosyncrasique d'images caractérisées par une opacité rémanente qui résiste à toute lecture linéaire, en appelant plutôt à un réseau de "lectures tangentes » dont l'apprentissage est lié à l'usage d'un faisceau disparate de métaphores qui agissent comme une espèce de médiateur idéoplastique des formes abstraites de l'analyse, tel qu'on peut le constater par exemple à la lecture du texte de l'Aufgabe. Ici encore, j'ai amplifié l'analyse proposée par Berman. Face à des tournures parfois assez byzantines et ce réservoir d'images qui brillent par leur opacité, il nous incombe, comme l'écrit Berman, "d'élucider non seulement le "labyrinthe" conceptuel qu'est la réflexion de Benjamin, mais d'illuminer les "images" qui l'émaillent " $(A T$, p. 28).

Ce qui nous entraîne directement au cœur de la poétique de Benjamin, c'est là le cinquième trait caractéristique de sa pensée : une prosodie heurtée qui opère un maillage très subtil entre un propos délibérément ésotérique, obscur, et une capacité d'illumination sans égale, qui perce l'écorce des phénomènes pour nous introduire dans le vortex où s'illumine l'essence même de la réalité, et ce tout en lui conservant, notamment en ce qui a trait à cette limpidité immédiate qu'il reconnaît au langage comme vecteur de la vérité arrachée aux diktats de la raison et du « sens 
commun », toute son obscure densité. Dans une missive adressée à Martin Buber, Benjamin écrit : "Si variées que soient les formes selon lesquelles le langage peut se montrer efficace, il ne l'est pas en communiquant des contenus, mais en produisant au jour de la manière la plus limpide sa dignité et son essence » (1979, p. 117). Berman qui, l'ai-je souligné d'entrée de jeu, est un pédagogue hors pair, explicite fort bien ce trait de sa pensée :

Magique est ce qui exerce un effet sans aucune médiation. Tous les textes de Benjamin sont écrits à ce niveau où le langage est magique, c'est-à-dire sans la médiation de raisons et d'éclaircissements. Ils traitent les concepts en les rendant d'abord opaques, c'est-à-dire en les soustrayant à la sphère des lieux communs. Dans un deuxième temps, ils les rendent plus parlants, ou plus illuminants. Benjamin emprunte presque toutes ses catégories à la tradition, mais les travaille de telle façon qu'elles deviennent pratiquement indécodables et hermétiques. Au sein de cet hermétisme, ses textes irradient. Benjamin était tout à fait conscient de ce trait de sa pensée. (AT, p. 29)

La poétique de Benjamin est organisée selon le modèle d'un "labyrinthe inachevé " dont la logique inextricable en son principe a pour but de révéler l'essence même des choses, d'en radiographier les formes pour produire leur teneur de vérité, alors que les mots sont nimbés d'une aura qui voile et illumine tout à la fois. L'illumination, le trait qui élucide et rend lucide, éclate sous la poussée de l'instant comme pure déhiscence de la forme fugitive celée sous la réserve de l'obscur ${ }^{4}$. Tenu pour cryptique par d'aucuns, le texte de l'Aufgabe, comme le souligne Berman, est effectivement une crypte, mais dans la mesure où "l'essence de la traduction s'y trouve à la fois montrée et cachée. Faire d'un texte sur la traduction une crypte celant son essence, telle était la manière pour Benjamin de correspondre à ce qu'il a appelé dans son doctorat sur le Romantisme allemand "la nature infiniment énigmatique de la traduction” » (AT, pp. 30-31). C'est pourquoi on ne saurait citer un segment de ce texte, le détacher de son ensemble sans se perdre dans un dédale qui confine à l'absurde,

4 Quoique je veuille m'en tenir le plus possible au commentaire de Berman et ne pas surcharger mon texte, je me permets de renvoyer sur ce point à l'excellent ouvrage de Margaret Cohen, Profane Illumination. Walter Benjamin and the Paris of Surrealist Revolution (1993). 
car sa poétique obéit à une dynamique non-linéaire qui génère une nébuleuse de thèses péremptoires, d'emblée contradictoires, qui, une fois isolées, s'effondrent et s'avèrent non analysables. Ce texte ne peut être cité, encore moins résumé, seulement commenté. Cette clause de lecture, sa postulation par Berman, est l'un des points forts de son commentaire, qui en fait une incontestable réussite sur le plan de l'interprétation.

Pour Benjamin, la traduction n'a rien d'une rubrique négligeable qui serait tout au plus ancillaire à la gestation originale des ouvres sous l'indicatif formel d'une prestation de sens liée à la transmission de contenus. Il entretient un triple rapport à la traduction. D'abord, en la sollicitant comme levier important de ses spéculations sur le langage et l'art. Ensuite, en tant que lecteur-de-traductions, qui constitue pour lui un acte sui generis ${ }^{5}$. Enfin, en tant que traducteur, que je qualifierais personnellement de "naufragé ", puisque lui-même il a pu constater assez vite son échec : sa génialité s'effondre lorsqu'il touche à la traduction et se dégonfle au profit d'une facture plutôt scolaire, sans grand relief (voir Janet Sanders, 2003). La vérité, c'est que la pratique concrète de la traduction s'est révélée pour lui un terrain miné, comme s'il ne savait négocier la part de vertige créatif et la dose d'audace requise pour un tel labeur, qui l'a amené à sombrer dans la platitude. Bref, la "pulsion-de-traduire», cette Übersetzungstrieb qu'évoquait Novalis dans une lettre à August-Wilhelm Schlegel, traducteur éminent s'il en fut ${ }^{6}$, lui faisait carrément défaut.

5 Je cite ici in extenso la note de Berman sur cette question : "La lecture-de-traductions est un mode fondamental de rapport-à-latraduction. L'expérience de la traduction n'est même pas pensable sans la lecture de traductions. Celle-ci devrait être un réquisit de tout enseignement de la traduction. Lire des traductions n'est pas simplement les rapporter à leurs originaux par voie de comparaison. C'est un acte sui generis » (AT, p. 31, note 34$)$.

6 C'est Berman qui nous reportait à cette remarque de Novalis dans l'ouvrage sur lequel il était encore penché lorsqu'il nous a quittés, Pour une critique des traductions : John Donne (1995, pp. 74-75, note 83): "C'est la pulsion-de-traduction qui fait du traducteur un traducteur : ce qui le "pousse" au traduire, ce qui le "pousse" dans l'espace du traduire. Cette pulsion peut surgir d'elle-même, ou être réveillée à elle-même par 
Nous sommes en présence d'un paradoxe qu'on peut considérer emblématique d'une démarche qui a tout pour désarçonner, puisque l'inverse est nettement plus fréquent : « nous avons ici un grand penseur de la traduction qui n'est pas un grand traducteur » (AT, p. 34).

L'abîme qui se creuse ici ne peut manquer de nous révéler quelque chose, du moins doit pouvoir être interrogé dans la trame du texte qui est censé introduire à une traduction, même ratée, des Tableaux parisiens de Baudelaire. Mais il ne parle en aucune façon de la matière dont il constitue le prologue. Tel une espèce d'aérolithe, de météore tombé du ciel que l'on aurait accolé à un matériau bien terrestre. $\mathrm{Ni}$ essai proprement dit, ni manifeste, ni théorie, ledit prologue accuse un hiatus abyssal avec la matière qu'il est censé introduire.

La tension nourrie par ce hiatus, Berman la rapporte à l'horizon platonicien de la traduction, qui en fait " un acte de translation transparente du sens » (AT, p. 36). Cet effacement, cette double auto-oblitération, de la traduction comme du traducteur, engendre une tension nodale qui départage et met en conflit la part de la traduction et celle qui serait dévolue au traducteur. On ne peut faire l'impasse sur la subjectivité du traducteur, qui marque toujours une négativité, le lieu même de son intervention, son momentum. Ce que tend précisément à oblitérer l'emphase mise sur la méthodologie dans le cadre de la tradition platonicienne de la traduction, qui commande la dissolution de l'agent traducteur, la mise au pas de sa subjectivité. En revanche, dès que le discours sur la traduction « cesse d'être "méthode", collant à un objet prédéfini, un hiatus s'ouvre entre la traduction (son expérience) et le discours sur la traduction. Ce discours ne peut ni la régir, ni l'éclairer. Et cela crée une tension et un paradoxe " (AT, p. 38). Il y a tension et paradoxe, en effet, car si nous pouvons nous prononcer sur l'exercice de la traduction à la faveur de principes " régulateurs " pour ce qui est de la "lettre ", ces principes ne peuvent ressortir à une méthode : « Entre l'espace du principe et l'acte de traduire existe un obscur espace de choix où interviennent

un tiers. Qu'est-ce que cette pulsion? Quelle est sa spécificité? Nous l'ignorons encore, n'ayant pas encore de "théorie" du sujet traduisant ». 
la subjectivité et l'inconscient. Le discours ne peut ni toucher à cet espace, ni explorer cette région où la traduction s'est accomplie dans ses mouvements essentiels. Il n'est donc pas homogène à l'expérience de la traduction» (AT, pp. 38-39).

Cette désolidarisation de toute prémisse méthodologique indique en creux que le propos de l'Aufgabe s'annonce comme une mise en question rigoureuse du paradigme platonicien de la traduction et qu'à l'appui d'un entrelacs assez noueux de cas de figures il compte approfondir ce hiatus et manifester avec force cette tension nodale qui nourrit et tient en suspens l'expérience de la traduction, mais il va le faire, paradoxe instructif, au nom d'un hyper-platonisme. De Charybde en Scylla.

Le titre du prologue laisse lui-même présager ce nœud gordien sous une tournure en apparence programmatique, qui annonce une redéfinition critique d'une responsabilité incombant au traducteur. Mais, comme le stipule Berman, le terme Aufgabe, dénotant une "tâche ", est mobilisé par Benjamin suivant une tout autre acception et sur un tout autre registre, qui n'a rien d'abord d'éthique. Il renvoie plutôt au " cosmos terminologique romantique » où la notion d'Aufgabe est immédiatement rapportée à un autre terme, l'Auflösung - "solution » ou " résolution »aussi bien solution au sens logique (d'un problème), que (dis) solution au sens chimique (d'une substance), ou encore (ré)solution au sens d'un accord musical. L'Aufgabe devient ici recherche d'une solution dans l'ordre du langage, plus précisément, comme l'écrit Berman, "une opération qui concerne la "dissolution" d'une "dissonance" primordiale dans la sphère du langage " $(A T$, p. 40). Dans ce cas, et c'est là le nœud du problème et la clef de sa " solution ", l'opération du traducteur n'est plus concernée par une finalité extérieure à l'exercice du langage lui-même, bref n'est plus liée par la loi implicite de la prestation d'un sens finalisé dans un acte de transmission ou de communication, mais est sollicitée par la «solution » immanente à la traduction en tant qu'elle touche à l'essence même de la vérité mise en œuvre dans l'ordre du langage : "Il y a ici re-définition de la "tâche" du traducteur, puisque nous sommes placés d'entrée de jeu dans une sphère qui n'a plus rien à voir avec le concept usuel de traduction comme opération de transmission » (AT, p. 41). 
Pas de doute que cet exercice préalable de décantation des lignes de force du microcosme benjaminien, la physionomie de sa pensée, esquissées ici à grands traits par Berman, est d'une indéniable fécondité et va nous permettre de suivre les linéaments et de scruter les arcanes d'une œuvre qui ne se laisse guère enfermer dans les lois du " genre ».

\section{Le commentaire}

\section{Cahier 2}

L'incipit du prologue, qui a fait couler beaucoup d'encre, notamment en ce qui a trait à l'autarcie (auto-référentialité) de toute œuvre d'art, comporte un triple volet formé d'une série d'affirmations d'une rigueur implacable : l'horizon du traduire est celui des œuvres : traduction-des-œuvres. Son essence se déduit de celle des œuvres. L'essence des œuvres n'est pas communication (AT, p. 46).

Nous avons donc une limitation implicite quant à l'objet de la réflexion de Benjamin, une détermination d'essence et une inférence percutante, péremptoire, sur la non-extension de cette essence, qui ne réfère qu'à elle-même, sui generis.

Il ne s'agit pas d'une réflexion sur la traduction " en général »: Benjamin s'attache exclusivement ici à la traduction des textes profanes (littéraires) et des textes sacrés, dont les sphères respectives ne sont pas étanches.

Une œuvre est toujours œuvre-de-langage et ne peut générer qu'une réflexion sur le langage, son essence. La rupture la plus radicale se situe au niveau de la conception largement partagée d'une œuvre comme instrument de communication véhiculant un message dirigé vers un destinataire :

[...] kein Gedicht gilt dem Leser, kein Bild dem Beschauer, kein Symphonie der Hörerschaf.

[...] aucun poème ne vaut pour le lecteur, aucun tableau pour le spectateur, aucune symphonie pour l'auditoire.

(AT, p. 47; trad. A. Berman) 
Le pôle de la réception se voit ici répudié sans ambages. Ce n'est pas rien. Benjamin nous dit que l'œuvre n'est pas tournée vers nous, c'est nous qui nous tournons vers elle. La traduction sera mise à contribution au profit d'une activité qui ne comporte aucune finalité extérieure à elle-même. Benjamin congédie du même coup la présumée relation d'intentionnalité qui pose tout objet pour un sujet qui le vise. Et dans la foulée de cette mise en orbite du pôle réceptif, c'est la prérogative de l'esthétique qui se voit dissoute et frappée de caducité. C'est un geste très radical et d'une grande portée pour ce qui est de saisir l'essence même du traduire et prendre la mesure de son oblitération séculaire au profit d'une " esthétique de la réception ", qui est presque un pléonasme. Berman note à juste escient : "C'est au nom du destinataire que, séculairement, ont été pratiquées les déformations qui dénaturent plus encore le sens de la traduction que les œuvres elles-mêmes. En fait, traduction ethnocentrique et traduction hypertextuelle se fondent sur une idéologie de la réception. En fait, la traduction ethnocentrique axée sur le lecteur transforme l'œuvre en message » (AT, p. 48).

Dans le paragraphe deux, Benjamin écrit :

Was «sagt " denn eine Dichtung? Was teilt sie mit? Sehr wenig dem, der sie versteht. Ihr Wesentliches ist nicht Mitteilung, nicht Aussage.

Mais que « dit » une œuvre littéraire? Que communique-t-elle? Très peu, à qui la comprend. Ce qu'elle a d'essentiel n'est pas communication, n'est pas énonciation.

(AT, p. 49; trad. M. de Gandillac)

Cette auto-référentialité absolue de l'œuvre dont le langage ne communique rien ni n'énonce quoi que ce soit est sans conteste un postulat hérité de la théorie critique élaborée dans la mouvance de la constellation post-romantique, laquelle entrecroise la «versabilité » (inter-traduisibilité des formes, mixité des " genres ", entre autres) infinie d'une écriture fragmentée, perméable à l'ironie et au Witz, et la Potenzierung, l'" élévation à la puissance » de l'œuvre qui fait valoir l'autonomie inconditionnée de toute poétique et célèbre ainsi son inachèvement paradoxal comme mode d'accomplissement absolu de son indice de finitude. Je module très fortement et $j$ 'accentue pour bien mettre en relief la condition dégagée par Berman : "Dans le poème, le langage 
s'accomplit purement en lui-même, mais à une condition : ne plus rien transmettre, ne plus rien communiquer, à la limite ne plus rien "signifier". Il est d'autant plus accompli qu'il se contente de reposer en lui-même. Et c'est ce langage-là, ce langage détourné de nous et de ses usages utilitaires, que rencontre le traducteur " (AT, p. 49).

Benjamin se lance aussitôt à l'assaut d'une prémisse un poncif pratiquement - monnayée par le "sens commun ", selon laquelle une traduction serait faite pour les lecteurs qui ne comprennent pas l'original. La question est :

Gilt eine Übersetzung den Lesern, die das Original nicht verstehen? Une traduction est-elle faite pour les lecteurs qui ne comprennent pas l'original?

(AT, p. 50 ; trad. M. de Gandillac)

Le translat proposé par Gandillac pour « gilt» est inexact, car le verbe gelten signifie "valoir ». Berman propose une autre leçon, mais ce qui est important ici c'est que le verbe gelten revient ailleurs dans le texte, car c'est l'un des termes de base qui constituent "le tissu lexical du texte en dessous de son tissu conceptuel » $(A T$, p. 50). Le verbe gelten, à l'instar du verbe verstehen, comprendre, comme du terme Ausdruck, expression, ou encore le concept de " traduisibilité ", d'Übersetzbarkeit, appartient au fuseau des Leitworter, pour user ici d'une notion introduite par Franz Rosenzweig (voir Lamy, 2003, pp. 204-206), bref des « mots recteurs » qui émaillent la portance du texte, qui en scandent littéralement le réseau conceptuel. La re-traduction de Berman est la suivante :

Une traduction vaut-elle pour les lecteurs qui ne comprennent pas l'original? ( $A T$, p. 51)

C'est une question lourde de sens, et pas seulement parce qu'elle entend visiblement fausser compagnie à une cause déjà entendue : une traduction est destinée à ceux qui ne comprennent pas la langue de l'original. Berman se livre ici à un exercice pédagogique tout à fait remarquable, que je vais tenter de radiographier dans ses grandes lignes. 
D'abord, à supposer que nous comprenions toutes les langues existantes, scénario peu plausible voisinant l'absurde, nous serions toujours en reste pour déterminer la nature de notre rapport à ce qu'on désigne comme un " original », ce qui est d'origine dans un " original ", puisque cette teneur ne peut que nous échapper. Par exemple, les koinai, ou langues de haute culture, médiatrices profuses et diffuses, sont toujours des langues-de-traduction, traduites et traduisantes, et, de ce fait, comme l'écrit Berman, « ont dès leur origine été entretissées de traductions » $(A T$, p. 52). Comment postuler un " pur » rapport de l'œuvre à sa langue alors que la fibre nerveuse de cette langue est tissée de traductions, bref le fruit d'une acculturation composite, immémoriale, tributaire de divers affluents idiomatiques exogènes?

Une œuvre ne parle jamais que dans sa langue, qui ellemême est saturée de vocables et de tropes empruntés à des constellations langagières limitrophes ou plus éloignées dans le temps et dans l'espace. Ainsi, comme nous le rappelle Berman, les cultures polylingues des $\mathrm{XVI}{ }^{\mathrm{e}}$ et $\mathrm{XVII}{ }^{\mathrm{e}}$ siècles traduisaient des œuvres qu'elles pouvaient déjà lire dans leur langue d'origine : la traduction permettait de « moduler» une œuvre. En l'occurrence, la " traduisibilité " d'une ouvre est aussi bien un indice de sa finitude, de son horizon d'origine, qu'ouverture à sa traduction, à ses multiples translations dans le microcosme des langues et des œuvres.

Tout ceci repose sur un paradoxe foncier, dont l'hypothèque ne peut être levée : "la communication n'est pas l'essence de la traduction, parce qu'une œuvre est intraduisible " (AT, p. 52). Berman explicite : "Toute expérience de l'acte de traduire l'atteste : rien de ce qui fait la chair langagière d'un texte (chair qui fait le vrai sens de l'œuvre) ne saurait être "transmis", car aucune équivalence réelle n'existe de langue à langue » $(A T$, pp. 52-53). Je condense l'argument : la " traduisibilité » d'une œuvre, l'appel qu'elle lance à son insu dans chacun des maillages de sa facture d'origine, est liée à sa migration dans "l'espace de l'intraduisibilité ». Ce qui signifie deux choses. D'abord, "la traduction n'est point recherche d'équivalences, mais mouvement vers la parenté des langues. Elle produit cette parenté sans la supposer »(AT, p. 53). Ensuite, toute œuvre est liée à sa 
langue sur un double mode, d'emblée contradictoire, mais qui appartient à l'essence même de sa manifestation : elle est aussi bien enracinement, plongée vers la racine, que dépassement, transcendance génératrice d'un écart dont l'amplitude se mesure précisément dans l'acte de traduire qui extrade l'œuvre et projette l'inachèvement paradoxal de sa facture définitive, l'intraduisible même, comme une matrice qui l'ouvre de l'intérieur en ménageant l'interface qui la cheville au " devenir des langues ", tout en révélant l'unicité et l'inviolabilité de sa « lettre »:

Par l'enracinement, l'œuvre s'enfouit dans l'épaisseur de la langue natale; par l'écart, elle s'arrache à elle en produisant une " autre " langue, étrangère dès lors à la langue commune. Enracinée, l'œuvre est intraduisible. Subversion de sa langue, elle l'est au plus haut degré. Et ici advient une curieuse dialectique. La traduction, d'abord, ne fait que radicaliser ce mouvement de subversion. Elle a pour « fin » de déporter l'œuvre toujours plus loin de sa langue. Mais plus une œuvre est traduite, plus s'accroît pour elle la possibilité de s'enraciner dans sa langue en apparaissant comme intraduisible. Cela n'apparaît guère au moment de sa " naissance ", où ce périple n'a pas encore été effectué. Elle n'apparaît comme œuvre-de-la-langue-natale que lorsqu'elle est (re)traduite. On peut donc dire que la traduction accomplit le rapport de l'ceuvre à sa langue. (AT, p. 53)

Benjamin y va ensuite d'un énoncé lapidaire :

\section{Übersetzung ist eine Form.}

Cette forme, qui est entendue au sens où Goethe l'a développée, celle d'un organisme possédant sa loi intrinsèque, qui est vecteur de métamorphoses, tient sa « loi » de l'original, qui n'est autre que sa " traduisibilité " - Denn in ihm liegt deren Gesetz als in dessen Übersetzbarkeit beschlossen (AT, p. 54).

La forme est « informée " par une loi qui lui est à la fois immanente, comme dans le vivant ou l'œuvre, et transcendante, comme dans la traduction qui trouve son principe dans la traduisibilité de l'original. Bref, la traduction surgit organiquement de l'original, qui encourt une métamorphose. Toujours en écho à Goethe, Berman explicite : « Le domaine des formes est celui des métamorphoses. La métamorphose est une auto-transformation 
de la forme. Celle-ci, en fait, n'existe que dans la métamorphose : c'est le "meurs et deviens" de Goethe » (AT, p. 55).

C'est un énoncé de principe, le pivot de la réflexion de Benjamin sur la traduction : dire que la traduction est une " forme ", c'est affirmer qu'elle est une métamorphose de l'original, et non une transformation extérieure à l'œuvre. Je pousse plus loin : la traduction n'est pas translation, déplacement d'un point $\mathrm{A}$ à un point $\mathrm{B}$, mais mutation endogène de l'œuvre exposée à un " milieu » exogène : transmutation ou, pour le dire dans les mots d'Haroldo de Campos : "transcréation ». Ce « milieu » exogène, langue ou culture-hôte, n'est pas la « cause » de cette métamorphose mais, si je puis dire, il la "précipite " (ici au sens où on en use en chimie : le " précipité » d'une réaction chimique, l'une des formes de l'Auflösung), la provoque en quelque sorte, alors que cette mutation appartient au développement intrinsèque, organique, de l'œuvre qui déploie ses potentialités latentes à la faveur d'une traduction dont la loi est précisément l'Übersetzungbarkeit de l'original' ${ }^{7}$ Ici je m'écarte brièvement du commentaire de Berman

7 Pour peu, je pourrais développer un modèle " darwinien » de la traduction, mais ce n'est évidemment pas le lieu pour le faire. J'observe sans plus que la migration des œuvres à travers les divers polysystèmes traductionnels exhibe des propriétés qui s'apparentent à une capacité d'adaptation et à une sélection " culturelle » qui font que certaines œuvres demeurent longtemps sous le boisseau ou connaissent une plus longue période d'incubation avant d'être traduites, tandis que d'autres se mettent à éclore soudainement dans la mouvance d'un " coup de dés " éditorial qui précipite la translation massive de leur corpus. Je me prévaux ici d'une brève incise pour nous reporter aux travaux d'Andrew Chesterman, un brillant pédagogue de la traductologie, qui, prenant appel sur le modèle poppérien de la théorie comme solution de problèmes, a développé une théorie des " mèmes » de la traduction (1997), dont l'idée est empruntée à l'éthologue britannique Richard Dawkins dans son fameux ouvrage The Selfish Gene et selon laquelle la culture, à l'instar des gènes en biologie, génère des entités " mimétiques " appelées memes par Dawkins et qui se reproduisent, se diffusent et tendent à s'incruster et à se perpétuer dans le champ culturel jusqu'à temps qu'elles soient supplantées par d'autres et soient ainsi vouées à l'obsolescence. Il en va des idées et des œuvres comme des gènes : il en est qui sont vouées à l'extinction ou à une bien brève prestation, et d'autres qui se répandent et prolifèrent par voie de duplication et d'adaptation évolutive dont 
et me permets de renvoyer à un passage absolument lumineux de l'essai de Benjamin, Sur le langage en général et sur le langage des bumains, rédigé sous forme de lettre qu'il fit parvenir de Munich en novembre 1916 à son ami Gershom Scholem et qui corrobore la leçon que je viens de proposer (je traduis) :

Die Übersetzung ist die Überfübrung der einen Sprache in die andere durch ein Kontinuum von Verwandlungen. Kontinua der Verwandlung, nicht abstrakte Gleichheits- und Ähnlichkeitsbezirke durchmisst die Übersetzung.

La traduction est le transfert d'une langue dans l'autre à travers un continuum de métamorphoses. La traduction traverse des continuums de métamorphose, non pas des zones abstraites d'équivalence et de ressemblance. (1991, p. 151)

Toute traduction, d'aussi lointain horizon puisse-t-elle poindre, participe d'un mouvement de transformation global des langues et des cultures qui, sous peine de se languir dans le plus plat chauvinisme et de se terrer dans un vase clos, sont intimement sommées de s'ouvrir à la fécondation de l'œuvre étrangère qui elle-même sollicite, à son insu, les auspices d'un épanouissement insoupçonné à la faveur d'une traduction obéissant à cette loi inhérente à l'œuvre, sa « traduisibilité ». Mais cette Übersetzbarkeit doit pouvoir trouver un point d'impact, un terreau où frayer sa voie, un canal et un moment propice à son éclosion. C'est l'enjeu de la prochaine question soulevée par Benjamin, qui est double, la première se voulant plus arbitraire et empirique, quoique décisive dans certains cas : celle du traducteur adéquat, tandis que la seconde évoque le "désir » immanent à l'œuvre et qui touche comme tel à son essence en relation à cette forme qu'est la traduction :

la traduction est certes un vecteur de prédilection. Cette perspective n'est pas étrangère au propos de Benjamin, car plus loin dans le texte il parle de la traduction comme la "survie " (Überleben) d'une œuvre, ou encore de sa Fortleben, une vie qui lui est octroyée de surcroît, ce qui est en continuité avec son acception goethéenne de la notion de " forme » comme matrice de croissance et vecteur de métamorphoses. J'y reviendrai ailleurs. 
[...] oder, und eigentlicher: ob es seinem Wesen nach Übersetzung zulasse und demnach — der Bedeutung dieser Form gemäß — auch verlange?

[...] ou bien, plus proprement : de par son essence admet-elle (zulasse) la traduction et - conformément à la signification de cette forme - la désire-t-elle (verlange)?

(AT, p. 55; trad. A. Berman)

Certaines œuvres n'ont l'heur d'être traduites que lorsque les temps s'y prêtent, sont mûrs pour les y accueillir. Facteur impondérable ou tournure du destin, nul ne saurait décréter sur des bases objectives quelles forces agissent pour qu'il en aille ainsi. Dans le fragment de Sens unique mis en épigraphe, Benjamin dit que le commentaire et la traduction se détachent de l'" arbre " du texte profane le moment venu, rechtzeitig. Ce qui évoque le mûrissement de l'œuvre, d'une part, et le désir de la langue traduisante, de l'autre. Le terme rechtzeitig appelle aussi la notion grecque de kairos, le pressentiment et la saisie du "moment opportun ». Comme le souligne Berman, le «possible » ouvert par le kairos de la traduction est lié à l'émergence d'un désir, j'ajouterais d'une "élection » qui pourrait ne pas survenir mais dont on peut pressentir la poussée, l'urgence. Ce désir appartient à la structure profonde d'une œuvre et détermine sa traduisibilité, son Übersetzbarkeit, mais aussi sa résistance à la traduction. C'est la vérité même du paradoxe selon lequel, écrit Berman, «le désir de traduire n'acquiert lui-même plénitude et signifiance qu'au contact des œuvres qui résistent le plus à la traduction, qui sont réputées intraduisibles ». Dans cette résistance, conclut Berman, « il y a un appel muet à la traduction. L'intensité de la résistance indique en creux que, pour l'œuvre, il y va dans la traduction de tout son être » $(A T$, p. 59).

Übersetzbarkeit. Avant de clore l'examen de ce Cahier, je me permets d'ouvrir une parenthèse pour souligner un trait caractéristique de la poétique de Benjamin, en l'occurrence l'usage du suffixe -barkeit, qui est associé chez lui à diverses modalités et configurations de sens au gré desquelles les œuvres aussi bien que les artéfacts culturels sont appelés à migrer et à se transformer, désignant une propriété inhérente à la composante intime de la dynamique à la faveur de laquelle ils ont vu le jour, mais qui n'est pas encore apparente au moment 
de leur gestation. Ce suffixe est récurrent chez lui et « aimante " en quelque sorte la conceptualité qu'il mobilise dans le tropisme de son écriture, la prosodie qu'il met à profit avec une maestria parfois déconcertante. Le rôle stratégique que joue ce morphème dans l'économie générale de la théorie critique élaborée par Benjamin m'est apparu encore plus clairement à la lecture du très bel ouvrage de Samuel Weber, Benjamin's -abilities (2008). Le faisceau de topiques qui alimentent son interprétation, fort pénétrante, a pour centre de gravité ce suffixe qui cheville le procédé heuristique de l'"illumination profane " que Benjamin met en œuvre dans une variété de contextes dont Weber examine les diverses déclinaisons, notamment impart-ibility, criticizability, cit-ability, translat-ability, reproduct-ibility. « Throughout his life ", écrit-il, "Benjamin tended to formulate many of his most significant concepts by nominalizing verbs, not in the usual manner but by adding the suffix -barkeit » (ibid., p. 4). Ainsi trouve-t-on déjà l'expression Mitteil-barkeit, " communicabilité ", dans son essai "Sur le langage en général et sur le langage des hommes »(2000c [1916], pp. 142-265), mais aussi la notion de Kritisier-barkeit dans sa dissertation sur Le concept de critique esthétique dans le romantisme allemand, qui sera publiée en 1920 (1986), évidemment le concept-clef d'Übersetz-barkeit dans l'essai qui nous intéresse de plus près ici (paru en 1923), mais encore celui de Reproduzier-barkeit dans son séminal essai, paru en 1935, sur « L'œuvre d'art à l'ère de la reproductibilité technique » (j’y reviendrai plus loin) et, enfin, la notion d'Erkenn-barkeit, de « cognoscibilité » ou celle de Les-barkeit, de " lisibilité » dans le Cahier " $\mathrm{N}$ » (Convolute $N$ ) des manuscrits préparatoires à la rédaction des Passages parisiens, pièce de résistance de son Nachlass, dont l'ensemble est connu aussi sous le titre Das Passagen-Werk, ou encore The Arcades Project dans l'excellente traduction anglaise qui en a été fournie (1999; v. également 1989).

Comme le stipule Weber, il ne s'agit pas simplement d'une simple idiosyncrasie stylistique. À mon sens, nous sommes en présence d'un opérateur modal de tout premier plan qui, à bien des égards, est symptomatique, à tout le moins anticipateur de l'ère où nous évoluons, laquelle, il va sans dire, loge largement à l'enseigne du virtuel (cyberespace, projection actuarielle, prospective, monnaie électronique, entités immatérielles, remix, matrices de duplication, CD-ROM). Il agit en quelque sorte, pour 
user d'un concept de la thermodynamique assorti aux propriétés émergentes de la complexité, comme une espèce d'" attracteur étrange " (strange attractor) qui polarise de manière diffuse les clusters thématiques, nébuleuses conceptuelles et constellations de sens où s'agglomère le très large spectre d'objets d'étude qui ont piqué la curiosité boulimique de Benjamin ${ }^{8}$. En y réfléchissant bien, cet usage prolixe du suffixe -barkeit m'a aussi rappelé l'assemblage de remarques lapidaires, parfois ésotériques, que nous a livrées le penseur italien Giorgio Agamben dans La comunità che viene, où il prend appel sur la distinction aristotélicienne de l'« être en puissance " (dynamis) et de l'« être en acte » (energeia) et ses diverses excroissances dans les débats autour de la grammaire spéculative élaborée par les doctes médiévaux ${ }^{9}$.

Or la posture critique de Benjamin le situe d'emblée sur le pôle de l'" être en puissance », de la pure virtualité. Un symptôme éloquent de cette option est sa dissension marquée à l'endroit de ce que Nietzsche désignait en gros comme l'« histoire des historiens ", qu'a remarquablement bien explicitée Michel Foucault, en l'occurrence une forme d'analyse historique qui en réalité annihile l'épaisseur stratifiée du temps tissé de contingences et de heurts de toutes sortes au profit d'une fresque peuplée d'acteurs idéalisés nous monnayant des vérités éternelles (voir Foucault, 1971, pp. 145-172; 1994, pp. 136-156). Mais ce ne

8 Benjamin était un collectionneur compulsif; voir à ce sujet Ursula Marx et al., 2007.

9 Voir G. Agamben (1990), qui nous rappelle, dans un premier temps, que pour les philosophes médiévaux « le passage de la puissance à l'acte, de la forme commune à la singularité, n'est pas un événement accompli une fois pour toutes, mais une série infinie d'oscillations modales. L'individuation d'une existence singulière, loin d'être un fait ponctuel, est une linea generationis substantiae qui varie selon une gradation continue de croissance et de rémission, d'appropriation et d'impropriété ». Plus loin encore, il ajoute : « Le passage de la puissance à l'acte, de la langue à la parole, du commun au propre, a lieu chaque fois dans les deux sens selon une ligne de scintillement alternatif où nature commune et singularité, puissance et acte échangent leur rôle et se pénètrent réciproquement. Lêtre qui s'engendre sur cette ligne est l'être quelconque, et la manière dont il passe du commun au propre et du propre au commun s'appelle usage - c'est-à-dire ethos » (pp. 25-27). 
fut guère une tâche facile, car c'est la contradiction majeure qui a été vécue par Benjamin, dans sa propre existence comme dans ses nombreuses tentatives pour s'expliquer avec la catastrophe anticipée que fut le $\mathrm{XX}^{\mathrm{e}}$ siècle. D'abord rompu à la doctrine des «pures essences» (platoniciennes), tel qu'il appert à la lecture de la matière passablement abstruse de la "Préface épistémo-critique " (Erkenntniskritische Vorrede) de son essai sur le Trauerspiel (1925), où l'essence même de la vérité configurée dans le nom qui en est l'épiphanie immédiate en sa pure puissance d'évocation en appelle à l'extinction de toute intention et nous situe hors du communicable ${ }^{10}$, son œil critique s'est aussitôt vu confronté, avec une constance accrue, à un amoncellement de ruines, la fragmentation stellaire de la réalité. Orphelin du royaume des " pures essences ", l'historien doit se faire " chiffonnier " et prospecter l'amas de rebuts, débris et reliquats à l'abandon sur le terrain laissé en friche par les vainqueurs de l'histoire.

10 Benjamin (1985) réintroduit avec force des éléments d'analyse qui couraient en filigrane de son essai de 1921 sur « La tâche du traducteur » : "La vérité est un être sans intentionnalité, formé à partir des idées. Par conséquent l'attitude adéquate, ce n'est pas de constituer une visée par le moyen de la connaissance, mais d'entrer et de disparaître dans la vérité. La vérité, c'est la mort de l'intention. [...] La structure de la vérité exige donc un être qui égale par son absence d'intentionnalité l'être simple des choses, mais qui lui serait supérieur par sa constance. La vérité ne consiste pas dans une visée qui trouverait sa détermination à travers la réalité empirique, mais dans un pouvoir qui donnerait d'abord sa forme caractéristique à l'essence de cette réalité. L'être détaché de toute phénoménalité qui seul a ce pouvoir en propre, est celui du nom. C'est lui qui détermine le caractère donné des idées. Mais celles-ci sont données moins dans une langue originelle que dans une perception originelle, où les mots possèdent le noble privilège de nommer, sans l'avoir perdu dans la signification, qui est liée à la connaissance. [...] L'idée est quelque chose qui relève de la langue, et plus précisément, le moment, dans l'essence du mot, où celui-ci est symbole. Dans la perception empirique, où les mots se sont dégradés, ils ont un sens profane manifeste à côté de leur aspect symbolique plus ou moins caché. C'est l'affaire du philosophe que de rétablir dans sa primauté, par la présentation, le caractère symbolique du mot, dans lequel l'idée se rend intelligible à elle-même, ce qui est à l'opposé de toute espèce de communication tournée vers l'extérieur » (pp. 32-33). 
La masse critique d'images et d'idées qui seule permet de reconfigurer la vérité pulvérisée dans l'accélération vertigineuse et la diffraction des phénomènes happés par le vortex qui converge au cœur du cône temporel ne peut être approchée qu'à la faveur d'une élaboration micrologique, dont Benjamin a partagé et disputé la notion avec Theodor Adorno, bref une prospection minutieuse qui fouit dans les interstices et les anfractuosités les plus infimes du symbole brisé, jusqu'à l'infinitésimal, le laissépour-compte dans les catacombes de l'histoire. Il s'agit alors, comme l'écrit Benjamin dans un segment du Passagen-Werk, de découvrir «dans l'analyse du plus petit moment individuel le cristal de la conjoncture globale ». Et dans une note préparatoire de ce même chantier, il précise encore : «La relation entre l'élaboration micrologique et les proportions d'ensemble montre comment le contenu de vérité (Wahrheitsgehalt) ne se laisse saisir que par l'immersion la plus rigoureuse dans les particularités du contenu des choses (Sachgehalt)" (cité dans Wohlfarth, 1986, p. 561). Cette investigation micrologique, qui est corrélée au coefficient d'" illumination profane " que recèlent les artéfacts les plus anodins de la culture matérielle, entraîne une démultiplication des perspectives dont l'unité - le « cristal de la conjoncture globale »- ne peut affleurer qu'à la faveur d'une conceptualité constamment réaménagée. Or, cette façon toute singulière qu'a Benjamin d'user d'une conceptualité qui consiste en un réordonnancement virtuel (virtuelle Anordnung) des constellations d'éléments parfois à l'abandon dans leur gravitation aléatoire, mais qui portent néanmoins la trace ou la signature du temps, ses cicatrices, se manifeste précisément - c'est le point que je désirais marquer - dans son procédé de substantivation des verbes sur le mode de la pure virtualité. Comme le suggère Samuel Weber, "This mode of conceptualizing "virtualizes" the process of nominalization by referring it back to what in German is very appropriately designated as a Zeitwort: a "time-word", aka verb, that is inseparable from time insofar as it involves an ongoing, ever-unfinished, and unpredictable process: Erkennbarkeit thus names the virtual condition of Erkennen, Benennbarkeit that of Benennen, Kritisierbarkeit that of Kritisieren, and so on " (op. cit, p. 7). 
$\mathrm{Si}$ nous approfondissons ce filon et en poursuivons la logique jusqu'à son ultime conséquence, il nous apparaît clairement - certes moins étonnant - que pour Benjamin, sans doute dans le sillage des intuitions développées par les Romantiques d'Iéna, l'œuvre n'est œuvre qu'en prêtant le flanc à de multiples métamorphoses ou, si je puis dire, à diverses -barkeiten qui sont autant de déclinaisons de son " être en puissance ", de sa Potenzierung : citabilité, criticabilité, traduisibilité, reproductibilité. Cette intuition est d'autant plus fructueuse qu'elle se révèle à mon sens tout à fait patente : que sont, en effet, les œuvres d'Homère, de Dante ou de Shakespeare en dehors de ces potentialités vectorielles? Je peux y répondre : le royaume de la literacy est un royaume de pure potentialité, et le coefficient de métamorphose qui lui est inhérent répond comme tel à la condition humaine, à l'essence de sa manifestation, qui demeure une équation insoluble ou à de multiples variables, la plupart inconnues. Le qualificatif « vectoriel » que j’y ai apposé signifie tout simplement que cette potentialité est inductrice de sens, sauf qu'elle ne dicte pas la direction et, a fortiori, la destination ultime du processus qui l'amène à exhiber des propriétés émergentes, virtuelles, qui la projettent dans des aires de jeu hétérogènes à sa source tout en conservant la trace de sa ligne d'univers, le signe de son origine se trouvant désormais dans le futur qui devient l'hôte d'une propension inhérente à l'œuvre qui la rend passible d'afficher telle ou telle autre propriété sous telle ou telle autre condition.

Cette série de -barkeiten qui affectent l'œuvre, formant une chaîne réflexive dont la diffraction prismatique reflue sur son noyau originel sans l'entamer, peut être interprétée, suivant le mot de Giorgio Agamben (op. cit.), comme « une série d'oscillations modales ». Mutatis mutandis, nous pourrions considérer que la vie de l'œuvre, tributaire de l'indice de futurition de l'Überleben et de la Fortleben prodiguées par les soins de la chaîne réflexive tissée au gré de ses diverses traductions, bref que l'individuation de son existence singulière, pour paraphraser à nouveau Agamben, « loin d'être un fait ponctuel, est une linea generationis substantiae qui varie selon une gradation continue de croissance et de rémission, d'appropriation et d'impropriété » (ibid., pp. 25-26). Derechef, il en va des œuvres comme de leurs traductions : s'appropriant à une 
époque où elle pourra aussi éventuellement être désappropriée par un coup du sort ou une soudaine désaffection de son lectorat, une œuvre peut pareillement être appelée à migrer par voie de traduction, à se voir transmutée, revitalisée et régénérée mais dans des formes qui, sans en altérer le noyau, la déportent au-delà de l'aire de jeu qui a hébergé sa prime gestation, et qui se déclinent précisément sous les diverses modalisations des -barkeiten: sa traduisibilité devient le levier de potentialisation de sa lisibilité, de sa citabilité, de sa criticabilité, de sa reproductibilité, voire de son adaptabilité intersémiotique (opératique, cinématographique et autres). Bref, l'œuvre ne s'approprie à elle-même, ne se conquiert en propre, n'acquiert son ethos, la loi de son usage propre, qu'au prix de multiples métamorphoses dont la traduction est l'un des canaux de prédilection.

Ce trait phénoménologique confirme l'une des propriétés inhérentes à l'exercice de la traduction, non pas tant sa "secondarité ", qui en ferait un "produit dérivé " ancillaire à l'original, mais plutôt, dirais-je, sa " postériorité », son " aprèscoup » et, par voie de conséquence, sa réflexivité immanente qui est marquée par un angle de déférence singulier, celui de la dissymétrie affectant l'interface entre des vernaculaires dont l'acte de naissance, la croissance et l'évolution exhibent les cicatrices et les sutures du temps, un faisceau de contingences, de métissages et d'hybridations de toutes sortes. Mais il corrobore aussi un trait comportemental de l'espace de la traduction qu'a fort bien mis en lumière Antoine Berman et qui, faute d'être érigé en norme, dénote une constante de son exercice, à savoir le fait que les "grandes traductions " sont toujours des retraductions (voir Berman, 1990, pp. 3-4; Gambier, 1994, pp. 413-417), lesquelles inscrivent un point d'orgue dans l'entropie des formes livrées aux avatars du temps, aux éphémérides d'une culture en proie à l'obsolescence et dont le renouvellement est souvent tributaire d'un apport significatif de l'étranger. Mais cette tangente conjoncturelle qui perméabilise les frontières imaginaires ou concrètes d'un terroir, nonobstant les facteurs convergents, est aussi empreinte de contingence et de volatilité, car elle en appelle à la saisie d'un kairos, du moment opportun où peut s'opérer la revitalisation de l'œuvre qui, une fois «transplantée » (verpflanzen) dans une contrée étrangère, pour reprendre un mot que Benjamin 
emprunte à Herder, pourra libérer des potentialités latentes, voire insoupçonnées, qui sauront féconder la culture-hôte.

D'aucuns ont cru bon s'objecter à cette inférence de Berman sur la base de quelques exceptions notoires. Or l'observation de Berman tient amplement la route à mon sens : non seulement parce que l'objection confirme la règle, mais parce que j'estime qu'il nous est loisible d'étendre l'enjeu et la portée de ce phénomène au-delà du seul processus de canonisation d'une œuvre au gré de son rayonnement et de la félicité de ses diverses greffes dans telle ou telle autre culture-hôte. Cette dynamique peut aussi entraîner dans sa mouvance la translation massive d'une nouvelle vision du monde, l'éclosion extraterritoriale d'un nouveau continent de la pensée dont l'acculturation parfois soudaine et accélérée à la faveur d'un cycle de traductions et de retraductions intensif, très concentré dans le temps, précipite l'intronisation subite, fulgurante, d'une façon de voir les choses qui élargit considérablement les horizons de la culture-hôte, quitte à en déliter l'assise.

Je songe ici aux multiples tractations traductionnelles, anthologiques, herméneutiques et autres qui ont mobilisé la fine fleur de l'intelligentsia allemande, à la fin du XVIII et au début du XIX ${ }^{\mathrm{e}}$ siècle, autour de la Bhagavad Gìtā, texte-culte du canon védique indien, qui fut reçu avec un luxe d'apparat critique et d'engouement par les jeunes zélateurs de la Bildung romantique, mais qui n'était en fin de compte que la pointe de l'iceberg dans le vaste océan de la littérature sacrée et de la métaphysique d'inspiration hindouiste ${ }^{11}$.

Le premier penseur allemand à se piquer d'un authentique intérêt pour la culture indienne et à se pétrir de la poétique de la Gìtā, est Johann Gottfried Herder, qui a pu alors s'en enquérir dans la traduction anglaise de Charles Wilkins, un éminent orientaliste associé à William Jones, le pionnier des études orientales en terre d'Albion. Sa version sera publiée à Londres en 1785 (Bhagrat-

11 Pour les développements qui vont suivre, voir la remarquable étude de Bradley L. Herling (2006); voir aussi John James Clark (1997). Pour ceux et celles qui seraient désireux d'approfondir ces questions, voir le très instructif recueil de Horst Turk et Anil Bhatti (1997). 
geeta, or Dialogues of Kreeshna and Arjoon, London, Nourse Publ., 1785 ) et sera ensuite traduite en français en 1787, et en allemand en 1802. Friedrich Schlegel, l'initiateur du cercle de l'Athenäum à Iéna, le fief des premiers Romantiques allemands, traduira les huit premiers chapitres de la Gìtā, dont on retrouve de larges extraits dans son séminal essai Sur la langue et la sagesse des Indiens (Über die Sprache und Weisheit der Indier, 1808), rédigé à la suite de son séjour à Paris où il s'était rompu à l'étude des langues orientales. Son frère, August Wilhelm, qui avait réussi à se procurer la police de caractères Devanagari, fabriquée à Paris, publia en 1823 sa propre traduction latine de la Gìtā avec le texte sanskrit en regard, constituant le tout premier ouvrage imprimé en sanskrit à voir le jour en Europe continentale. Wilhelm von Humboldt, qui étudia le sanskrit auprès du linguiste Franz Bopp, proposera sa propre traduction allemande de la Gìtã en 1826. Hegel, farouche promoteur de l'idéalisme allemand, produira aussitôt un compte rendu très critique du commentaire de Humboldt, dépréciant avec vigueur les vertus de la culture védique qu'il estimait inapte à se hisser au niveau du Concept guidant l'Esprit dans son odyssée dialectique pour conquérir l'identité absolue entre l'être et le devenir. Arthur Schopenhauer, nihiliste désabusé et héraut du désenchantement, fera de la Gìtā sa lecture de chevet, qu'il dégustait à petite dose chaque soir avant de plonger dans le nirvana prodigué par les soins de Morphée.

Il n'est pas négligeable d'observer que Walter Benjamin est un héritier direct de cette tradition de pensée, mais cette équipée traductionnelle est autrement riche d'enseignements. Un constat éloquent, et non des moindres, est ce trait caractéristique de la culture-hôte, qui n'est certes pas exclusif à la Bildung allemande, se révélant promptement encline à faire de la Bhagavad Gìtā une espèce de surrogat biblique, comme si la culture indienne devait nécessairement, elle aussi, être en possession d'un livre " révélé ", à l'instar de la civilisation judéo-chrétienne. On constate aisément que même chez des esprits passablement cultivés, de mentalité libérale et gratifiés d'une sensibilité d'une grande plasticité, baignant alors dans un « bouillon de culture » à l'affût des horizons les plus lointains, il demeure difficile de s'affranchir d'une Weltanschauung profondément ancrée, de parvenir à se dépayser suffisamment, à se doter d'une réserve prudentielle sur 
l'universalité présumée de ses propres assises, pour clairement et librement percevoir l'altérité de l'Autre, l'étranger.

La Bhagavad Gìtā, dont le topique est l'enseignement de la loi du dharma, l'assignation d'un destin auquel on ne peut échapper, est devenue une œuvre-phare de la Weltliteratur et un vade-mecum des âmes éprises de spiritualité, mais elle n'a rien d'un livre "révélé ». C'est une pièce de littérature, certes célébrée dans son terreau natal et qui peut être conçue comme un abrégé de la doctrine védique, mais la Gìtā demeure une portion congrue enchâssée dans le vaste cycle de chants épiques recueillis dans le Mahäbhärata. Elle a connu un sort similaire au Tao Te King (Dao De Jing), attribué à Lao tseu (Laozi), que les premières traductions de la vague " orientaliste " européenne ont infléchi et corseté dans une livrée transie des embruns éthérés d'une mystique de l'ineffable, alors que les examens plus scrupuleux de la paléographie sinologique de pointe, jouissant évidemment d'un éventail plus copieux de spécimens se prêtant à une collation plus affine, nous révèlent un texte destiné à parfaire l'éducation du prince et à lui prodiguer les préceptes d'une gouvernance sereine et sans faille, assurée par la vacuité impassible de la voie du milieu, le vide médian qui est le principe même du Dao.

Tout ceci me rappelle une remarque judicieuse - elle est de Theo Hermans je crois, je cite de mémoire-, selon laquelle l'examen des traductions nous en apprend davantage sur les traducteurs, leurs commanditaires ou les instances qui les sollicitent ainsi que sur la culture-hôte en général, que sur le texte-source lui-même. Puisque nous y sommes, je crois qu'il importe de comprendre que l'essai de Benjamin, aussi bien que le commentaire de Berman ou celui, du reste, que je propose ici, ne concernent pas exclusivement la traduction comme telle, dans son fonctionnement, mais aussi, de façon expresse, sa capacité d'exhiber les mécanismes profonds de transformation de la perception du monde. Comme j'aurai l'occasion de le réitérer, la traduction nous offre une lucarne privilégiée nous ménageant un poste d'observation qui nous habilite à scruter les rouages délicats qui gouvernent la mise en discours de l'expérience, décloisonnant les horizons de référence de cette expérience dans le contact avec l'étranger sur la ligne de front du langage. La traduction est mise à l'épreuve de notre 
horizon de référence, d'abord parce qu'elle nous confronte à ses limites dans l'expérience de cette "inquiétante étrangeté ", cette Unheimlichkeit qui est parfois vivement ressentie en sollicitant les ressorts de notre propre langue qui, dans la recherche d'une solution de traduction, semble soudainement se dérober à nous, elle qui pourtant nous apparaissait d'un usage si courant, d'un commerce si familier. C'est que, précisément, la traduction fait apparaître l'être-langue de la langue. À une échelle plus globale, la traduction nous baille accès aux mœurs migratoires de la pensée, tandis qu'elle prête son concours à la perméabilité transfrontalière de diverses économies symboliques dont l'entrelacs est tissé de nœuds de résistance comme de zones franches dédouanant les formes du microcosme langagier de manière à établir les « vases communicants » à la faveur desquels peut s'engager la cohabitation des cultures et des visions du monde. Ces considérations, loin d'être banales, sont brûlantes d'actualité (voir Apter, 2005, pp. 12-22). Car, comme le suggère le titre sélectionné à juste escient par les éditrices du séminaire d'Antoine Berman, nous vivons précisément à l'« âge de la traduction ". Les frontières sont en train de s'ouvrir. Malheureusement, la frontière la plus rigide, la plus étanche, se situe dans notre tête, dans notre perception de l'altérité de l'autre homme, le lointain comme le premier venu.

La perspective que je viens d'esquisser à grands traits modifie du tout au tout les paramètres à l'aune desquels nous jaugeons ce que nous désignons ou concevons comme une tradition littéraire ou culturelle. En bref, il n'est de tra-dition sans tra-duction, ce qui veut dire qu'une tradition ne peut vivre et se perpétuer, voire, au demeurant, naître et prendre forme qu'en se transformant : sa constance et sa fécondité résident dans la métamorphose de ses formes. Son noyau, inviolable, vit du métabolisme qui la renouvelle au gré de ses diverses mutations épidermiques. C'est une métaphore que nous allons retrouver plus loin sous la plume de Benjamin avec l'image du fruit, de son noyau et de sa peau. De plus, ces observations renforcent une idée que je vais introduire plus en aval, selon laquelle la spéculation benjaminienne est largement teintée d'un caractère proleptique, se déployant sur le mode de l'anticipation, qui recoupe l'arrière-plan messianique qui imprègne son propos. 


\section{Cahier 3}

Seconde séance du séminaire. Berman asserte à nouveau avec force la pertinence de la pratique du commentaire qui doit en fin de compte aménager le terrain, préparer le chantier en vue d'une re-traduction où converge le faisceau de "lectures tangentes » à l'approche du seuil marquant le stade critique de la plongée au cœur du texte. Cette plongée est motivée par l'attention portée au texte, l'« amour-de-la-lettre ». D'où la gémellité originaire entre philo-logie et philo-sophie, à l'instar de la traduction dont Franz Rosenzweig disait dans L'écriture et Luther quelle est un hieros gamos, fusion nuptiale ou noces sacrées entre deux langues (1998, p. 63).

$\mathrm{Au}$ début du paragraphe quatre, Benjamin serre de plus près ce qu'il entend par " traduisibilité ", en l'occurrence il départage le désir de l'ouvre et celui de la traduction : que la «traduisibilité » soit propre à telle ou telle autre œuvre, ne signifie pas qu'elle soit en souffrance de traduction, mais « qu'une signification déterminée, inhérente à l’original, s’exprime dans leur traduisibilité » ( $A T$, p. 67; trad. A. Berman). Mais une traduction, s'empresse-t-il d'ajouter, aussi accomplie se veuille-t-elle, ne signifie absolument rien pour l'original, qui se maintient, inviolé, dans son intégrité originelle. C'est l'une des assertions de Benjamin qui m’avait incité en 1997 à infléchir la dénotation standard du terme Aufgabe, "tâche ", vers la notion d'" abandon ", option sans doute risquée mais qu'avait ensuite entérinée mon collègue Alexis Nouss. Berman, pour sa part, explicite : « Le rapport de l'œuvre à ses dérivations traductives et critiques est de désir, mais ce désir est traversé d'ironie. L'ironie consiste en ce que, dans son être-plein, dans son être-achevé, elle relativise, par le simple fait quelle est, les traductions et les critiques auxquelles manquent éternellement plénitude et achèvement» $(A T, \mathrm{p}$. 68). Ce hiatus est néanmoins révélateur de la dignité de la traduction comme acte, tout en scellant le sort du résultat qui peut en découler, l'ironie étant dirigée sur son caractère purement aléatoire et adventice eu égard à l'autarcie absolue de l'original : « Ce que l'œuvre appelle de toutes ses forces, pour que la signification immanente à sa traduisibilité s'actualise, c'est bien l'acte de la traduction. Mais ce quelle considère avec une indifférence ironique, comme si cela 
ne la concernait en rien, c'est la traduction comme résultat» $(A T$, p. 68$)^{12}$.

Le distinguo opéré ici par Berman, judicieux, me rappelle l'observation décisive de Wilhelm von Humboldt, selon laquelle le déploiement de la langue, tant du point de vue de la parole proférée dans un acte singulier de signification que dans l'ampleur organique de ses multiples déclinaisons, est « non pas un ouvrage fait (ergon), mais une activité en train de se faire (energeia $)^{13}{ }^{13}$. C'est pourquoi l'original demeure inentamé dans son " noyau " le plus profond, que Berman associe à la pure musicalité, l'intraduisible même, point de résistance ultime dont l'œuvre de langage doit dompter l'irrépressible puissance d'attraction pour conserver sa parlance et sa signifiance (AT, p. 70). En effet, écrit Berman, c'est « le noyau de parlance ou de signifiance d'une œuvre qui doit être intraduisible (parce que quelque part cet intraduisible peut se renverser en traduisible), non son noyau de musicalité qui représente l'intraduisibilité absolue d'une dimension opaque, muette et insignifiante dans sa vague infinité » $(A T$, p. 70$)$.

Le maillage originel du traduisible et de l'intraduisible dans l'œuvre est insoluble, inextricable comme tel. Si la pulsion du traduire est à ce point sollicitée c'est que la traduisibilité l'y intime à la frontière de l'intraduisible car, nous dit Benjamin, la traduction se tient avec l'original, grâce à sa traduisibilité immanente, pourtant bordée d'intraduisibilité, "dans la plus

12 La facture originelle du Macbeth de Shakespeare restera à tout jamais indifférente à la superbe livrée dans laquelle Michel Garneau nous l'a présentée il y a trois décennies déjà, tel « un royal manteau aux amples plis »; en revanche, quoique je ne puisse en aucune façon deviner par quels processus de métamorphose implicites Garneau en est arrivé à sa « solution » de traduction, il est clair qu'il n'avait pas à se plier à ce que pouvait signifier et comment pouvait résonner pour les contemporains du grand Barde élisabéthain ladite facture originale, pour autant qu'on puisse le savoir car nous, la postérité, n'avons guère accès à ce qui se passe en « temps réel », à l'Urszene, la « scène primordiale » où s'est engagée la gestation de l'un des canons du polysystème littéraire mondial.

13 Voir W. von Humboldt (1974); les termes ergon et energeia sont des notions appartenant au lexique de la physique aristotélicienne. 
étroite co-relation " (im nächsten Zusammenhang). J'extrapole et pousse plus avant la glose de Berman : pareille sollicitude, qui peut prendre la forme d'une injonction, ne peut opérer qu'en marquant et en accentuant - la révélant en quelque sorte - la distance qui fonde cette co-relation originaire et la projette dans l'espace de résolution de la différence comme différer œuvrant dans l'amplitude de la vie comme historicité, non pas la vie au sens biologique, mais comme champ de l'expérience humaine, nourrie d'engendrements et de mûrissements dont la traduction est partie prenante. J'ouvre une brève parenthèse pour observer que la traduction est, à mon sens, un vecteur exemplaire de ce que Jacques Derrida désigne par le terme "différance » : toute œuvre diffère en elle-même, et elle diffère d'elle-même, malgré elle, et elle œuvre toujours malgré elle en " différé » car sa déférence à son point d'impact éventuel est indécidable, tout comme l'est la déférence de ce point d'impact putatif à la source qui s'y manifeste en creusant une béance qui, dans le cas qui nous occupe ici, tient la décision du traduire en son suspens, qui la précipite tout en marquant le retrait de l'original dans l'étanchéité originelle de sa forme qui néanmoins est fatalement appelée à se métamorphoser. C'est un peu ce que Benjamin nous distille ici avec sa série d'énoncés lapidaires et cryptiques. C'est pourquoi aussi, je le crois, cette co-relation originaire de la traduction et de l'original est d'emblée problématique : féconde et ingrate. Progéniture ingrate certes, mais le géniteur, l'original, l'a déjà rendue orpheline, sevrée, affranchie jusqu'à l'indifférence. En effet, nous dit Benjamin, « cette co-relation est d'autant plus intime qu'elle ne signifie plus rien pour l'original : Ja, dieser Zusammenhang ist um so inniger, als er für das Original selbst nichts mehr bedeutet » (AT, p. 72; trad. A. Berman $)^{14}$.

Cette co-relation ou entre-appartenance originaire de l'original et de sa traduction, qui pourtant ne signifie rien pour le premier alors que le second y trouve la détermination même

14 Je reproduis ici l'importante remarque de Berman sur l'enjeu et la portée du terme innig en allemand : "Innig se traduit par "intime". Mais ce mot allemand est un intraduisible. Car "l'intime" français est irrémédiablement subjectif et "intimiste". Innig, en allemand, renvoie précisément à cette entre-appartenance des êtres que désigne, entre autres, Zusammenhang. C'est une catégorie ontologique » (AT, p. 72). 
de son essence, sa ratio essendi, est « co-relation de la vie »Zusammenhang des Lebens :

So wie die Äußerungen des Lebens innigst mit dem Lebendigen zusammenhängen, ohne ihm etwas zu bedeuten, geht die Übersetzung aus dem Original hervor.

Tout comme les expressions de la vie co-appartiennent (zusammenhängen) au plus intime (innigst) avec le vivant (das Lebendige) sans signifier quelque chose pour lui, la traduction surgit (hervorgeht) de l'original.

(AT, p. 75; trad. A. Berman)

Le terme-clef ici est le verbe allemand hervorgebt, qui dénote la provenance à partir d'une origine, le jaillissement soudain. Dans ce « surgissement » il y a quelque chose de fortuit, d'imprévisible, comme une pure prodigalité, une excroissance insoupçonnée, qui se prodigue de surcroît et qui implique une relation d'inconnu, un peu comme dans une niche écologique le degré de variabilité affiché par les mutations auxquelles se prêtent les espèces n'est jamais totalement prévisible et n'affecte en rien la perpétuation du vivant qui aurait pu tout aussi bien emprunter d'autres voies. Pour l'original, qui se maintient dans son intégrité originelle, il n'y va pas tant de sa vie que de sa " survie ": "Zwar nicht aus seinem Leben so sebr denn aus seinem 'Überleben' " (AT, p. 76). Berman note que Benjamin met expressément le terme Überleben entre guillemets, car il y a co-appartenance, Zusammenhang, avec Übersetzung. La pensée allemande, des Romantiques d'Iéna à Heidegger, s'est révélée très prolixe au sujet du terme über, préposition souvent mobilisée comme préfixe, qui appelle à une élévation, un surmontement, tout comme chez Heidegger, l'Überwindund de la métaphysique, son dépassement, ne peut se produire qu'à la faveur d'un saut hyperbolique, dans la mouvance d'une ouverture abyssale ménagée pour l'écoute et le regard par le geste de tra-duction, l'Über-setzung, où la pensée accède d'un bond à la parole " augurale » des Grecs, le matin de la pensée. Benjamin nous entraîne, quant à lui, vers le « tardif », le « tard venu » de la traduction :

Ist doch die Übersetzung später als das Original und bezeichnet sie doch bei den bedeutenden Werken, die da ibre erwäblten Übersetzer niemals im Zeitalter ihrer Entstehung finden, das Stadium ibrer Fortlebens. 
Car la traduction est plus tardive (später) et caractérise pour les oeuvres importantes (bedeutenden), qui ne trouvent jamais (niemal) leur traducteur élu (erwäblten) à l'époque de leur naissance (Entstehung), le stade de leur vie continuée (Fortlebens).

(AT, p. 77 ; trad. A. Berman)

L'Überleben des grandes œuvres, nous dit Benjamin, leur " survie ", est pour ainsi dire "supplémentée » et, à la rigueur, rendue possible par une Fortleben, que Berman traduit ici par "stade de leur vie continuée ». Cette phrase comporte une gradation ponctuée d'éléments significatifs, à mon sens fort éloquents. Je re-traduis donc, à mon tour, à ma façon : le "tardif » (spät) n'est pas que l'effet d'un étalonnage dans la durée, opérant dans un continuum isomorphe, mais la retombée d'un " différé " qui caractérise structurellement la courbe de vie des œuvres "significatives »(bedeutenden) qui jamais (niemal) ne trouvent leur traducteur de prédilection (erwäblten) au moment de leur gestation (Entstehung), c'est pourquoi leur échoit une Fortleben, comme une vie de surcroît.

Cela signifie en outre que la vie des grandes œuvres se déploie sur un mode temporel propre, une ligne d'univers dotée d'une historicité singularisée qui distancie les conditions initiales de leur éclosion et les auspices sous lesquels la postérité est disposée à les accueillir. C'est une dynamique propre au destin des œuvres, qui ne présuppose aucune solution de continuité entre leur gestation et les critères qui d'aventure pourraient baliser et avaliser leur réception à une époque donnée. Aléas et augures d'une félicité promise au déclin accompagnent un rayonnement inattendu, parfois hors du commun, que Benjamin appelle « gloire » $(\mathrm{Ruhm})$. Mais ce « corps de gloire » n'a rien d'une épiphanie qui se projette dans l'éternité d'un instant. Comme le note Berman, " la survie de l'œuvre n'est nullement la pérennité d'une splendeur immobile ». Il nous reporte à la réflexion de Goethe, toujours d'une exaltante lucidité, pour qui justement «la traduction advient à l'œuvre comme destin et nécessité au temps de son rayonnement le plus radieux qui est aussi celui de son déclin» (AT, p. 82). Goethe va donc en tirer la conclusion qui s'impose : pour l'œuvre figée dans sa gloire, qui ne tardera à s'étioler, car elle est aussi annonciatrice de son déclin, la traduction est " régénération ", « rajeunissement ": Verjüngung. 
La traduction, en effet, apparaît comme " ce mouvement qui, en posant l'œuvre dans un ailleurs langagier, la libère de cette végétation étouffante, de l'immobilité intangible de sa propre gloire et - littéralement - la transplante dans un sol plus neuf, où elle se régénère. Oui : la traduction est transplantation" (AT, pp. 82-83, en italique dans l'original).

Je me permets d'observer que l'idée de la traduction comme "transplantation " (Fortpflanzung) était une métaphore fort en vogue chez les penseurs allemands inspirés, de près ou de loin, par les travaux de naturaliste de Goethe. Je l'ai repérée notamment chez Herder qui écrit : "Nous recevons des contrées étrangères des notions à transplanter dans notre langue - bier bekommen wir Begriffe aus fremdem Gegenden in unsre Sprache verpflanzen" (cité dans Pénisson, 1992, p. 164). Derrière cette métaphore consacrée, tirée du lexique du botaniste, se dessine en creux un impératif d'une grande portée, en l'occurrence un correctif de taille apporté à une perception encore assez courante et qui départage le mouvement vivant des formes à travers la traduction d'une idée préconçue qui la ravale à un rôle d'estafette ou de courroie de transmission. Loin de se résumer à la transmission d'un contenu vers une autre aire langagière, translation d'une langue à une autre, ou encore, comme l'écrit Benjamin, à une simple médiation (Vermittlung), la traduction est vecteur de métamorphoses et levier d'une réactivation des ressorts originaires de l'œuvre qui, à l'heure où sa gloire sonne le glas de son règne et voit son aura se dissiper pour entamer son déclin, est soudainement ramenée par les soins d'une traduction opportune au « temps de son surgissement ». Dans une traduction digne de ce nom, écrit Benjamin, « la vie de l'original atteint son développement constamment renouvelé le plus tardif et le plus étendu (umfassendste, littéralement : le plus englobant, embrassant) - In ibnen erreicht das Leben des Originals seine stets erneute späteste und umfassendste Entfaltung "(AT, p. 84; trad. A. Berman). Qu'est-ce à dire sinon que l'Übersetzbarkeit propre à l'œuvre, sa traduisibilité, est structurée par le mouvement de temporalisation qui ménage sa phase de maturation et l'acquisition de son autonomie propre comme forme de vie, laquelle est sanctionnée par un kairos, la saisie d'un temps opportun qui sollicite les offices d'une traduction qui saura à son tour se saisir de l'œuvre comme 
" un être vivant qui a son temps propre, un temps où elle est mûre pour la traduction » $(A T$, p. 85$)$.

\section{Cahier 4}

La traduction est un fruit tardif de l'œuvre promise à un constant renouvellement au gré des constellations langagières où elle atteint son "déploiement »(Entfaltung) de la plus grande amplitude (umfassendste). Le paragraphe cinq du prologue mesure l'enjeu et la portée de ce grand charroi qui déporte l'œuvre hors de son terreau natal :

Diese Entfaltung ist als die eines eigentümlichen und hohen Lebens durch eine eigentümliche und hohe Zweckmäßigkeit bestimmt.

Ce déploiement en tant que celui d'une vie originale (eigentümlich) et élevée (hohe) est déterminé par une finalité originale et élevée.

(AT, p. 87; trad. A. Berman)

Benjamin se lance ici dans une digression proprement philosophique qui associe à l'essence de la vie une finalité transcendante. Pour lui, il n'est de vie qui ne soit histoire, qui constitue son assise la plus vaste, matrice des grands cycles temporels où les formes vivantes œuvrent en vue d'une finalité supérieure. On songe évidemment au " règne des fins » au sens kantien. Tout ceci est problématique : ne trouvant pas sa fin en soi, toute manifestation de la vie est traversée par une finalité qui la transcende et qui relativise sa propre occurrence au profit d'une essence qu'elle a pour but d'exprimer, pour la « présentation de sa signification » (für die Darstellung seiner Bedeutung) :

Alle zweckmäßigen Lebensercheinungen wie ibre Zweckmäßigkeit überhaupt sind letzten Endes zweckmäßig nicht für das Leben, sondern für den Ausdruck seines Wesens, für die Darstellung seiner Bedeutung.

Tous les phénomènes vitaux doués de finalité, ainsi que leur finalité, possèdent au bout du compte une finalité non pour la vie, mais pour l'expression de son essence, pour la présentation de sa signification.

(AT, p. 89; trad. A. Berman) 
Mutatis mutandis, la traduction se voit confier un mandat qui procède de même source et la met à pied d'œuvre pour la Darstellung de la signification immanente à l'œuvre, qui demeure enfouie en elle, à l'état latent, comme " germe » d'une langue encore inaudible, aux échos infinis. La traduction n'est pas transmission d'un sens, quel qu'il soit, il lui incombe plutôt de "présenter ", de rendre présent quelque fragment ou éclat tardif de "la manière dont l'œuvre est signifiable dans sa totalité indépendamment des sens qu'on peut lui découvrir empiriquement. L'œuvre est captation d'une signification, d'une Bedeutung, infinie, inanalysable, insondable » ( $A T$, p. 91). Cette finalité est immanente à l'œuvre, mais lui est aussi étrangère en ce qu'elle ne peut, dans sa facture originelle, la déployer dans toute son ampleur et toute son intensité. Mais là n'est pas la question. Benjamin change abruptement de registre, nous déporte du plan de l'œuvre vers celui des langues qui, en dépit de leur désarmante disparité, sont nouées par un "rapport caché " (verborgene Verhältnis) que la traduction a pour finalité d'exhiber en un point de tangence furtif - parenté immémoriale qui affleure à peine dans cette progéniture tardive qu'est la traduction :

So ist die Übersetzung zuletzt zweckmäßig für den Ausdruck des innersten Verbältnisses der Sprachen zueinander.

Ainsi la traduction a-t-elle en dernier ressort pour finalité l'expression du rapport le plus intérieur (innersten) entre les langues.

(AT, p. 92; trad. A. Berman)

L'Innigkeit, catégorie ontologique de prédilection de la pensée romantique allemande, qui évoque une plongée vertigineuse au cœur de cette relation insigne, dans son intériorité la plus profonde, règne sur ce rapport, car il noue d'un lien nuptial intimité et distance, ampleur et intensité :

Sie kann dieses verborgene Verhältnis selbst unmöglich offenbaren, unmöglich herstellen; aber darstellen, indem sie es keimbaft oder intensiv verwirklicht, kann sie es.

Elle ne peut pas elle-même révéler ce rapport caché, ne peut pas elle-même le produire (herstellen), mais le présenter, en le réalisant en germe (keimhaft) ou intensivement, elle le peut.

(AT, p. 92; trad. A. Berman) 
Cet énoncé énigmatique est d'une incroyable densité. Chaque mot porte, comme l'écrit Berman, qui nous rend explicite l'un des pivots de sa compréhension : en l'occurrence le verbe herstellen, " produire ", qui prend le relais du verbe darstellen, " présenter". Il s'agit non pas de restituer ce rapport caché, mais de le produire, car "ce rapport caché n'est pas quelque chose de déjà existant " (AT, p. 93). Or cela, justement, la traduction ne le peut pas, il lui appartient pour l'essentiel de présenter cette relation en la « réalisant » (verwirklichen) en germe ou intensivement : keimhaft oder intensiv. La traduction annonce quelque chose d'absolument inédit qu'elle ne peut produire comme tel, mais qu'elle préfigure et vers lequel elle converge.

Le mandat est octroyé : dans le vaste royaume de la vie qui n'est autre que l'histoire où se déploie le champ entier de l'expérience humaine, la traduction intervient comme point ou plan d'effraction furtif, intangible, d'un rapport caché, immémorial, qui lie toutes les langues les unes aux autres, rapport qu'elle ne peut que "présenter " sur un mode intensif et fragmentaire. Berman rapproche ce keimhaft, en germe, d'une expression utilisée aussi par Benjamin à propos de l'œuvre selon Goethe : torsenhaft, en torse, à la manière du torse, ce qui ne va pas sans rappeler la fragmentation stellaire, la " pollinisation " constellée des formes poétiques et plastiques chez Novalis et Schlegel. Mais c'est là une propriété intrinsèque de la traduction : une gestation fragmentaire, rivée à l'inachevé, à l'éclat, la brisure. Rappelons-nous cette formule remarquable de son essai sur Les affinités électives de Goethe, citée plus en amont, où Benjamin écrit : "N'achève l'œuvre que d'abord ce qui la brise, pour faire d'elle une œuvre morcelée, un fragment du vrai monde, le débris d'un symbole » (1971, p. 234; 2000, pp. 274-395).

La traduction œuvre toujours sous le signe de l'inachevé, c'est pourquoi aussi son facteur de prédilection est le multiple: combien de traductions ont été laissées en plan, vouées à l'abandon? Esquissées, abandonnées, retrouvées, retravaillées, mises à nouveau sous le boisseau, et parfois complétées avec la vive conviction du défaut, de la carence, du reste insoluble, vestige d'un monde à jamais révolu. Mais aussi cendres ardentes sous la braise qu'un patient labeur nourri par l'amour de la lettre saurait 
réinsuffler. La traduisibilité de l'œuvre appelle une multiplicité de traductions : telle traduction précoce forme un fragment qui est " relevé » (au sens de l'Aufhebung hégélienne) par une retraduction formant un autre fragment et un autre encore traçant le profil embryonnaire d'un organisme vivant dont la généalogie finira par tisser une constellation nucléée par un vortex qui de spirale en spirale nous reconduit en cette intime anfractuosité où affleure l'augure furtif, fulgurant et bien éphémère éclat de ce « rapport caché » entre les langues.

Qu'est-ce à dire sinon que la traduction est toujours au banc d'essai? C'est précisément par le terme Versuch, " essai ", que Benjamin calibre le travail de la traduction, « le germe de sa production " (den Keim seiner Herstellung) qui est " un mode de présentation tout à fait original " (ein ganz eigentümlicher Darstellungsmodus) qu'on ne peut guère rencontrer dans «le domaine de la vie non langagière » (im Bereich des nicht sprachlichen Lebens) (AT, p. 94; trad. A. Berman). Novalis et Schlegel se prévalaient aussi fréquemment du terme Versuch pour qualifier le mode d'expérimentation qui caractérisait l'ensemble des stratégies mises à contribution pour activer la Potenzierung, l'" élévation à la puissance » des procédés poétiques. Cette excroissance tardive, fruit mûr tombé de l'« arbre " du texte profane, nous projette sur la ligne d'horizon d'un futur indéfini, où l'œuvre ne nous attend pas, car elle est immune, parfaitement achevée dans son inachèvement, intacte de toute déférence à sa progéniture, tandis qu'elle encourt une métamorphose qui porte au jour une signification latente qu'elle recélait à son insu. La traduction est un index de "futurition ", elle pointe en direction d'un à-venir en germe dans la forme achevée de l'œuvre. Si Benjamin parle ici de « réalisation » (Verwirklung), celle-ci opère essentiellement sur le mode de l'anticipation - anticipation de ce qu'elle ne peut que "présenter » : le lien intime entre les langues. D'où le chiasme (X) formé par une double temporalité enchevêtrée aussi bien que promise à la poursuite de parallèles qui ne se touchent qu'au point de fuite de cet horizon indéfini où la courbe du déclin qu'entame l'œuvre dans son terroir croise le coefficient d'anticipation du traduire qui, à travers le prisme de sa temporalisation propre, ménage une interface pour la « survie » de l'œuvre et l'aspiration à l'intime convergence des langues: 
Jenes gedachte, innerste Verbältnis der Sprachen ist aber das einer eigentümlichen Konvergenz.

Mais ce rapport pensé et très intérieur des langues est celui d'une convergence originale.

(AT, p. 96 ; trad. A. Berman)

Cette convergence est unique et eigentümlich parce qu'elle ne ressortit pas au jeu de ressemblances qui assortissent les langues naturelles dans des configurations arborescentes permettant d'en cartographier l'évolution. Benjamin écarte donc ici toute considération génétique, telle qu'on en trouve chez Humboldt par exemple, et qui repose sur une lecture a posteriori des phénomènes alors que, lui, il postule en quelque sorte un lien d'essence, bref il vise directement un critère ontologique fort, qui lie le dissemblable et n'a cure des ressemblances puisqu'il fait de cette dis-semblance même le lien qui apparente a priori toutes les langues dans leur mode de visée :

Es besteht darin, daß die Sprachen einander nicht fremd, sondern a priori und von allen historischen Beziehungen abgesehen einander in dem verwandt sind, was sie sagen wollen.

Elle [l'intimité des langues] consiste en ceci que les langues ne sont pas mutuellement étrangères, mais a priori, et indépendamment de toutes les relations historiques entre elles, sont parentes (verwand) en ce qu'elles veulent dire.

(AT, p. 96; trad. A. Berman)

Ce was sie sagen wollen, "ce qu'elles veulent dire ", sera transposé plus loin dans le texte dans le "mode de visée " (Art des Meinens) spécifique à l'œuvre et que la traduction doit avoir souci de réactiver et d'embrasser dans cette véritable opération d'obstétrique - Benjamin parle des « douleurs de l'enfantement » dans la langue traduisante qui vaque à la " post-maturation " (Nachreife) du vocable étranger - où elle doit quérir la forme qui atteste de la parenté immémoriale des langues. Un peu plus loin dans le texte aussi, nous le verrons, Benjamin va évoquer l'idée d'une ressemblance non sensible en relation avec le frayage intempestif de la reine Sprache, cette " pure langue " qui affleure furtivement en concertant les harmoniques disséminées dans le « devenir des langues » au point de convergence du cône temporel qui s'actualise dans l'interrègne entre original et traduction. Très subtilement se met en place une conception traditionaliste de la 
traduction, d'obédience essentialiste, puisque Benjamin fait valoir et postule en quelque sorte un lien d'essence qui apparente a priori la diversité des vernaculaires qui ornent le microcosme humain. Je crois que Berman avait raison de parler plus haut, d'entrée de jeu, d'une contestation paradoxale de la tradition platonicienne, mais au nom d'un byper-platonisme. Dès le début de cette dernière séance les dés étaient jetés lorsque Benjamin affirmait que le "déploiement » (Entfaltung) le plus tardif et le plus étendu de l'œuvre à travers la traduction est déterminé par " une finalité originale et élevée » (eine eigentümliche und hohe Zweckmäßigkeit).

Il y aurait matière à débat, car j'ai vraiment maille à partir avec cette idée d'une finalité transcendante qui déterminerait a priori aussi bien le processus de traduction que l'exercice du langage en général. Mais il n'est pas lieu d'en disputer ici ${ }^{15}$. Il y a

15 Je ne m'attarderai pas longtemps là-dessus, sauf pour préciser qu'à mon sens en matière de traduction il ne saurait y avoir ni relation de causalité stricte ni visée téléologique ou injonction d'une finalité, pour la simple raison que les langues sont des entités contingentes, même si bon nombre de leurs configurations obéissent à une nécessité interne de construction. Du point de vue ontologique, ce sont des « formes de vie » correspondant à une économie symbolique historiquement constituée dont les composantes affichent une haute teneur de contingence liée à divers facteurs d'acculturation et de métissage. Que l'allemand ou le français ou l'anglais soient ce qu'ils sont ne relève d'aucune nécessité et par conséquent leur inter-traduction ne peut obéir à un schème causal ou téléologique. La signification est modelée sur l'usage, comme le sont pareillement les règles grammaticales, qui sont découvertes après le fait, le fruit d'une investigation menée et conclue a posteriori. C'est du reste ce qu'avait déjà fermement établi César Chesneau Du Marsais (1676-1756) qui, dès 1729, affirmait : " La grammaire n'est pas avant les langues. Il n'y a point de langue qui ait été faite sur la grammaire; les observations des grammairiens doivent être faites sur l'usage "; et, en 1751, à l'article «Abstraction » de l'Encyclopédie, on pouvait lire : «Il n'y a pas hors de nous un être réel qui soit la grammaire; il n'y a que des grammairiens qui observent " (cité par Daniel Droixhe, 1978, p. 287). Je dirais, sans plus, que nous sommes simplement dans le domaine du pur " événement » : il en va de la vie comme des œuvres qui, à leur heure, peuvent avoir l'heur d'être avalisées par une traduction qui leur sied. Mais la relation d'inconnu demeure toujours prégnante car les œuvres sont aussi des « formes de vie » jouissant d'une autonomie propre. 
un point plus crucial à soulever, qui concerne la nature de l'objet sous examen. Je vais mécarter brièvement du commentaire.

Au début du séminaire, Berman a bien montré que sousjacente au propos de Benjamin se trouvait une métaphysique du langage. Il y a moyen d'être plus précis : derrière tout cela, en fait, il y a une mystique. Je dirais que c'est précisément à ce stade du texte que l'arrière-plan mystique qui imprègne la vaste majorité des écrits de Benjamin commence à infiltrer les veines de cette prosodie d'une désarmante efficacité, qui opère par touches sciemment parsemées au fil de métaphores dont la plasticité baigne dans le clair-obscur où l'évanescence diffuse des concepts le dispute à l'hyperlucidité des images tracées en creux et enchâssées à la façon d'une poupée gigogne. Le tout est cohérent, mais d'une cohérence baroque, puisque chacune de ses affirmations amorce un point de rupture et projette sa différentielle dans l'intégrale d'une forme qui configure la tradition dont elle émane. Je connais bien cette tradition : son propos s'inscrit dans la lignée de la doctrine des Noms divins dont l'origine est à jamais perdue et sur laquelle du reste pèse un interdit, à l'instar du tétragramme YHWH qui est réputé imprononçable : il n'est possible d'en approcher l'essence insondable qu'à travers la convergence des lignes brisées et des configurations à géométrie variable formées par la multiplicité des langues.

Ce nominalisme mystique cheville chacune des articulations du prologue de Benjamin, qui est un plaidoyer en faveur du caractère sacré du langage saisi dans l'angle de déférence et l'inachèvement du texte profane que la traduction ne peut « relever » ou « rédimer » qu'en se tournant vers l'épiphanie fugitive de la "pure langue » filtrant à travers les mailles de la forme qui s'y actualise. Derechef, la cohérence du texte n'est pas en cause, mais baroque elle l'est certes, comme je l'ai suggéré, puisqu'il est dénué de toute argumentation, nous monnayant plutôt une série d'assertions en cascade qui donnent parfois, à la limite, dans la pétition de principe. Le ton est péremptoire, ce qui lui confère une saveur oraculaire. Pour moi, c'est un peu comme l'envers symétrique du Tractatus logico-philosophicus de Wittgenstein, qui 
loge à la même enseigne ${ }^{16}$. Wittgenstein a produit un appareil logique saturé de propositions qui s'enchaînent avec une rigueur implacable pour nous révéler au terme de l'exercice que tout cela n'est que pur non-sens et un tissu de tautologies, car l'essentiel se trouve ailleurs, dans l'éthique et le mystique, ce dont on ne peut parler. Il va sans dire que ni l'un ni l'autre ne sauraient trouver grâce aux yeux de Karl Popper et satisfaire au critère de réfutabilité (ou de falsifiabilité) qu'il a postulé, selon lequel n'est valide et viable qu'une proposition qui est susceptible d'être réfutée. Or tout ce qu'avance Benjamin dans son prologue sur «La tâche du traducteur » est irréfutable. C'est pourquoi aussi, comme l'a affirmé Berman, ce texte est indépassable et, ajouteraisje, impraticable: il dessine de l'intérieur les limites auxquelles est confronté tout traducteur. Poursuivons.

(Fin de la première de trois parties.)

\section{Laurent Lamy Université de MontréAL}

\section{Références}

AGAMBEN, Giorgio (1990). La communauté qui vient. Théorie de la singularité quelconque. Trad. Marilène Raiola. Paris, Seuil.

APTER, Emily (2005). “Translation After 9/11: Mistranslating the Art of War". Dans The Translation Zone: A Nerw Comparative Literature. Princeton, NJ, Princeton UP, pp. 12-22.

16 Plus haut, lorsque Benjamin écrivait que la traduction ne peut révéler (offenbaren) et pas davantage " produire » (herstellen) le rapport caché qui apparente les langues entre elles, mais seulement le " présenter " (darstellen), cela m'a fait songer à la distinction cruciale chez Wittgenstein entre le « dire » et « montrer ", selon laquelle précisément la proposition montre la forme logique de la réalité, mais elle ne peut la dire, la révéler. Je me permets de citer ici cet énoncé lapidaire, d'une extrême concision, où l'on remarquera le très bel effet de symétrie, car Wittgenstein était à coup sûr un très grand styliste de la prose allemande : "Was gezeigt werden kann, kann nicht gesagt werden: Ce qui peut être montré ne peut être dit» (1960, p. 33). 
BENJAMIN, Walter (1966). «Über Sprache überhaupt und über die Sprache des Menschen ». Dans Angelus Norus, Ausgewählte Schriften 2. Frankfurt am Main, Suhrkamp Taschenbuch, p. 20.

BENJAMIN, Walter (1972). «Einbahnstrasse ». Dans Tilman Rexroth, dir. Gesammelte Schriften, Bd. IV/1. Frankfurt am Main, Suhrkamp Verlag, pp. 83-148.

BENJAMIN, Walter (1979). Correspondance, tome I. Trad. Guy Petitdemange. Paris, Aubier.

BENJAMIN, Walter (1985). Origine du drame baroque allemand. Trad. Sibylle Muller (avec le concours de André Hirt), préface de Irving Wohlfarth. Paris, Flammarion.

BENJAMIN, Walter (1986 [1920]). Le concept de critique esthétique dans le romantisme allemand. Trad. Philippe LacoueLabarthe et Anne-Marie Lang. Paris, Flammarion.

BENJAMIN, Walter (1988). Sens unique, précédé de Une enfance berlinoise. Trad. J. Lacoste. Paris, Maurice Nadeau.

BENJAMIN, Walter (1989). Paris, capitale du XIX siècle. Le livre des passages. Trad. Jean Lacoste. Paris, Éd. du Cerf.

BENJAMIN, Walter (1991). «Über Sprache überhaupt und über die Sprache des Menschen ». Dans RolfTiedemann et Hermann Schweppenhäuser, dirs. Gesammelte Schriften, Bd. II/1, Frankfurt am Main, Suhrkamp Verlag, pp. 140-157.

BENJAMIN, Walter (1999). The Arcades Project. Trad. Howard Eiland et Kevin McLaughlin, basé sur l'édition critique établie par Rolf Tiedemann. Cambridge, MA et Londres, Harvard UP.

BENJAMIN, Walter (2000). «Les Affinités électives de Goethe ». Dans Euvres I. Trad. M. Gandillac, revue par Rainer Rochlitz. Paris, Gallimard, pp. 274-395.

BENJAMIN, Walter (2000b). « Sur le concept d'histoire ». Dans Euvres III. Trad. M. de Gandillac, revue par Pierre Rusch. Paris, Gallimard, p. 427-443. 
BENJAMIN, Walter (2000c [1916]). « Sur le langage en général et sur le langage humain ». Dans Euvres I. Trad. M. de Gandillac, revue par Rainer Rochlitz. Paris, Gallimard, pp. 142-165.

BERMAN, Antoine (1990). « La retraduction comme espace de la traduction ", Palimpseste, 4, " Retraduire ». Paris, Publications de la Sorbonne Nouvelle, pp. 1-7.

BERMAN, Antoine (1995). Pour une critique des traductions : John Donne. Paris, Gallimard.

BERMAN, Antoine (2008). L'Âge de la traduction. "La tâche du traducteur " de Walter Benjamin, un commentaire. Texte établi par Isabelle Berman avec la collaboration de Valentina Sommella. Saint-Denis. Presses Universitaires de Vincennes, coll. «Intempestives ».

CHESTERMAN, Andrew (1997). Memes of Translation: The Spread of Ideas in Translation Theory. Amsterdam et Philadephie, John Benjamins.

CLARK, John James (1997). Oriental Enlightenment: The Encounter Between Asian and Western Thought. Londres et New York, Routledge.

COHEN, Margaret (1993). Profane Illumination. Walter Benjamin and the Paris of Surrealist Revolution. Berkeley, CA, University of California Press.

DAWKINS, Richard (1976). The Selfish Gene. Oxford, Oxford University Press.

DU MARSAIS, César Chesneau (1978 [1751]). «Abstraction ». Dans Daniel Droixhe. La linguistique et l'appel de l'histoire (16001800). Genève et Paris, Droz, p. 287.

FOUCAULT, Michel (1971). « Nietzsche, la généalogie, l'histoire ». Dans Hommage à Jean Hyppolite. Paris, PUF, pp. 145172. 
FOUCAULT, Michel (1994). " Nietzsche, la généalogie, l'histoire ». Dans Dits et écrits : 1954-1988, vol. 2, 1970-1988. Paris, Gallimard, pp. 136-156.

GAMBIER, Yves (1994). « La retraduction, retour et détour ». Meta, 39, 3, pp. 413-417.

HERLING, Bradley L. (2006). The German Gita: Hermeneutics and Discipline in the German Reception of Indian Thought, 17781831. Londres et New York, Routledge.

HUMBOLDT, Wilhelm von (1974). « La différence de construction du langage dans l'humanité et l'influence qu'elle exerce sur le développement spirituel de l'espèce humaine ». Dans Introduction à l'cuvre sur le kavi et autres essais, traduction et introduction de Pierre Caussat. Paris, Seuil, p. 133-420.

LAFONT, Cristina (2002). The Linguistic Turn in Hermeneutic Philosophy. Cambridge, MA, MIT Press.

LAMY, Laurent (2007). "Brève archéologie de l'hypothèse relativiste et du comparatisme linguistique dans la mouvance de l'Aufklärung ». TTR, 20, 2, pp. 9-62.

LAMY, Laurent (2003). « De la traduction comme vecteur clandestin de la rédemption : Franz Rosenzweig ou l'utopie messianique en l'absence de Dieu ». TTR, 16, 1, pp. 185-214.

MARX, Ursula, Gudrun SCHWARZ, Michael SCHWARZ et Erdmut WIZISLA, dirs. (2007). Walter Benjamin's Archive. Trad. Esther Leslie. Londres et New York, Verso.

PÉNISSON, Pierre (1992). Johann Gottfried Herder. La raison dans les peuples. Paris, Éd. du Cerf.

ROSENZWEIG, Franz (1998). "L'Écriture et Luther ». Dans L'écriture, le verbe et autres essais, traduction, notes et préface de Jean-Luc Evard. Paris, PUF, p. 55-82. 
SANDERS, Janet (2003). « Divine Words, Cramped Actions: Walter Benjamin - an Unlikely Icon in Translation Studies ». TTR, 16, 1, pp. 161-183.

SONTAG, Susan (2002). Under the Sign of Saturn. New York, NY, Picador US, Macmillan.

TURK, Horst et Anil BHATTI (1997). Kulturelle Identität: deutsch-indische Kulturkontakte in Literatur, Religion und Politik. Göttinger Beiträge zur internationalen Übersetzungsforschung, Bd. 15. Berlin, Erich Schmidt Verlag.

WEBER, Samuel (2008). Benjamin's -abilities. Cambridge, MA et Londres, Harvard UP.

WITTGENSTEIN, Ludwig (1960). Schriften. Frankfurt am Main, Suhrkamp Verlag.

WOHLFARTH, Irving (1986). " Et Cetera? De l'historien comme chiffonnier ». Dans Heinz Wismann, dir. Walter Benjamin et Paris. Colloque international, 27-29 juin 1983. Paris, Ed. du Cerf, p. 559-609. 